\title{
Exploration of novel geometric imperfection forms in buckling failures of thin-walled metal silos under eccentric discharge
}

\author{
A.J. Sadowski ${ }^{1}$ and J. Michael Rotter F.ASCE ${ }^{2}$
}

\begin{abstract}
The unsymmetrical discharge of a granular solid from a thin-walled cylindrical metal silo is well known to be a potential prelude to catastrophic buckling failure. The mechanics of this structure have only been very slowly unraveled in recent years with the help of powerful nonlinear finite element analyses. The associated buckling collapse is now known to be caused by localized axial membrane compression and occurs under predominantly elastic conditions. However, such high compressive stress concentrations are also known to produce significantly less imperfection sensitivity than uniform compression. For this reason, the search for an appropriately detrimental imperfection form under eccentric discharge has been quite a long one.
\end{abstract}

This study presents and explores a novel form of long-wave imperfection using a superellipse to parametrise the entire shell geometry. This imperfection, termed 'superelliptical flattening', is judged to be potentially present in practical silo construction, and is shown to cause significant decreases in the nonlinear buckling strength of an imperfect slender silo under eccentric discharge.

\section{Keywords}

Thin-walled metal silos, eccentric discharge, imperfection sensitivity, superellipse, nonlinear computational analysis, buckling failure.

\author{
Affiliations \\ ${ }^{1}$ Research Associate, The University of Edinburgh, Scotland, UK \\ ${ }^{2}$ Professor of Civil Engineering, The University of Edinburgh, Scotland, UK
}


Published in: International Journal of Solids and Structures, 50, 781-794.

DOI: http://dx.doi.org/10.1016/j.ijsolstr.2012.11.017

\section{Introduction}

Non-symmetric flow of the stored granular solid in thin-walled metal silos is a welldocumented critical design condition and a major cause of catastrophic buckling failures. The design challenge presented by this granular solids flow pattern, known as eccentric discharge, is exacerbated by the difficulties in both measuring and modeling the great range of possible flow patterns (e.g. Jenike, 1967; Hampe, 1991; Rotter, 2001a; Chen et al., 2007), each of which may have different structural consequences. The present study is concerned with buckling predictions for slender metal silos under a specific flow pattern known as eccentric pipe flow (Rotter, 2001a; EN 1991-4, 2006) (Fig. 1). There is extensive evidence that this flow pattern has the most damaging effect on the buckling strength of thin-walled metal silos (Rotter, 1986, 2001b; Wallner, 2010a and b; Sadowski, 2010; Sadowski and Rotter, 2010, 2011a, b; 2012a, b).

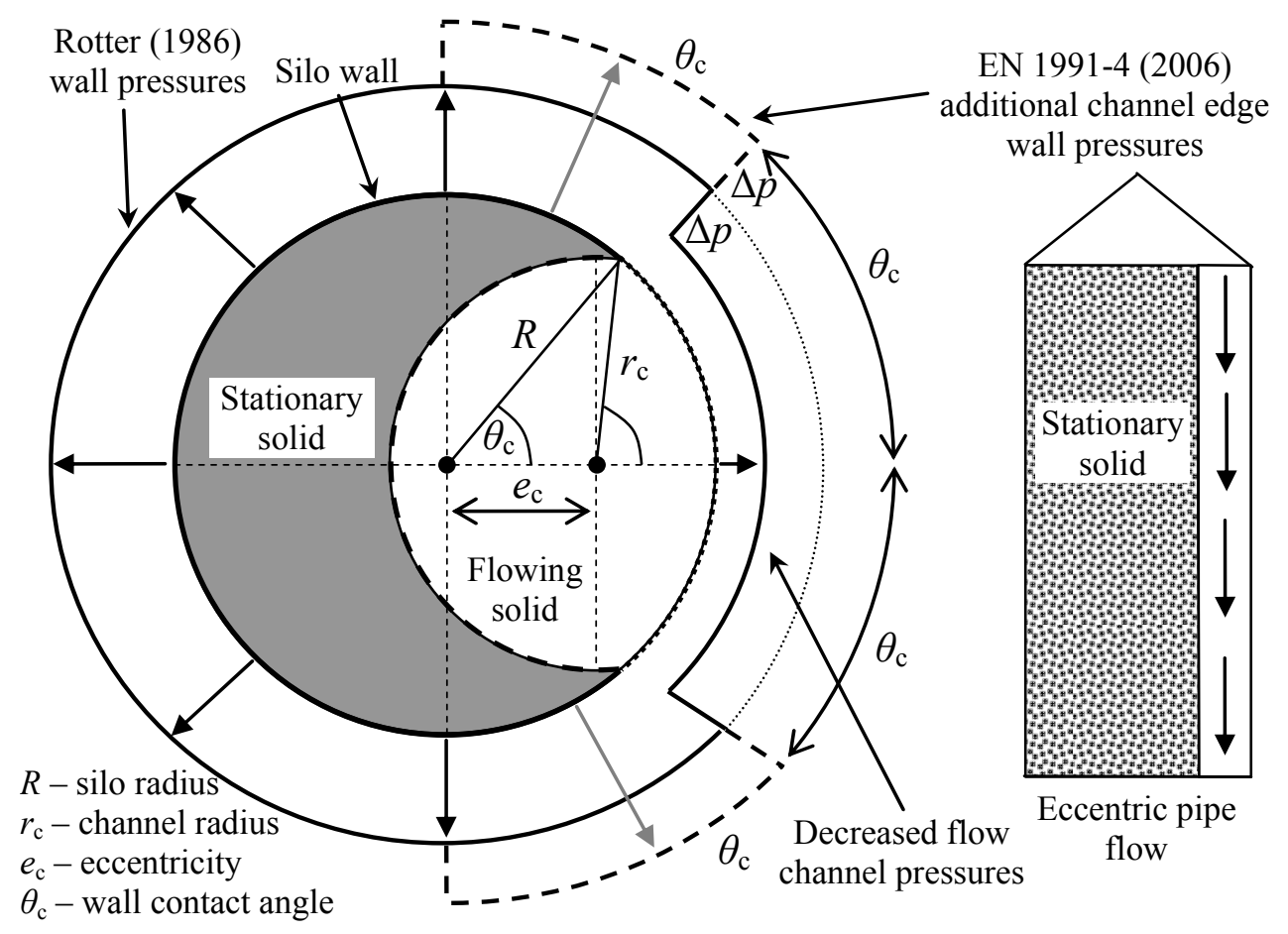

Fig. 1 - Outline circumferential distribution of normal wall pressures in a slender silo under eccentric pipe flow, based on geometry of Rotter (1986)

In the first description of eccentric pipe flow, Jenike (1967) considered a cross-section through the silo and assumed that only a limited portion of the stored solid flows 
during discharge. He assumed a flow zone in the form of a truncated circle of fixed geometry, with the remaining granular solid stationary. This basic geometrical arrangement, with more freedom in the form of the circular flowing zone, coupled with the assumption of a parallel-sided flow channel, was used by Rotter (1986) to derive a pair of differential equations governing the vertical distribution of wall pressures within the static and flowing solids. A typical prediction (Fig. 2) shows a significantly lower wall pressure within the flow channel and an increase in wall pressure beyond the reference Janssen value within the static solid. The pressures vary as a function of the flow channel radius $r_{\mathrm{c}}=\rho R ; \rho \in[0,1]$.

A simplified version of the above analysis is used in the European Standard on Actions on Silos and Tanks EN 1991-4 (2006). In this simpler version, the pressure in the stationary solid is set to the reference Janssen value regardless of channel size, and there is an additional flow channel edge local rise in pressure adjacent to the flow channel (Fig. 2). The existence of such a rise is based on experimental observations (e.g. Gale et al., 1986; Nielsen, 1998; Chen et al., 2007), but the magnitude chosen for EN 1991-4 (2006) appears to exceed any known measurements (Sadowski \& Rotter, 2012b). The formulations both with and without flow channel edge pressure rises are considered in this study. 


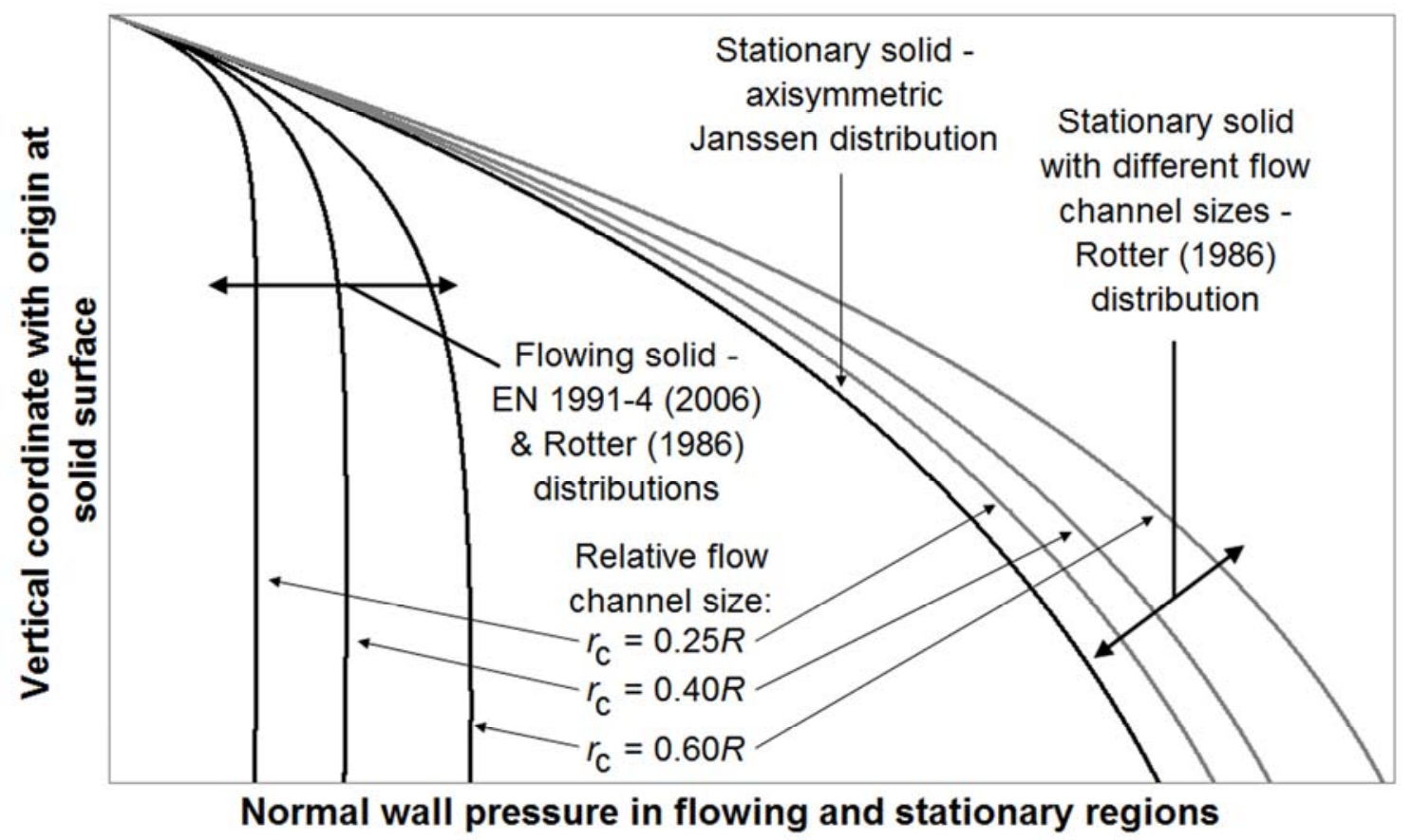

Fig. 2 - Outline meridional distributions of normal wall pressures in a slender silo under eccentric pipe flow

\section{Related structural studies of slender silos under eccentric pipe flow}

\subsection{Stress patterns and critical buckling locations in the perfect shell}

Previous structural studies of perfect slender thin-walled metal silos under the preceding formulation of eccentric pipe flow pressures (Rotter, 1986; Rotter, 2001b; Sadowski \& Rotter, 2010; 2011a, b; 2012a, b) revealed a characteristic distribution of axial membrane stresses and associated buckling behavior (Fig. 3). These studies investigated custom-designed example silos with optimized stepwise-varying wall thickness distributions, where the bottom of each strake was critical for buckling under concentric discharge conditions. Any one of the above references can give an appropriate background to the material presented here, including details of structural design and finite element modeling techniques used. These details are not repeated here to permit more directly useful new information to be presented. 


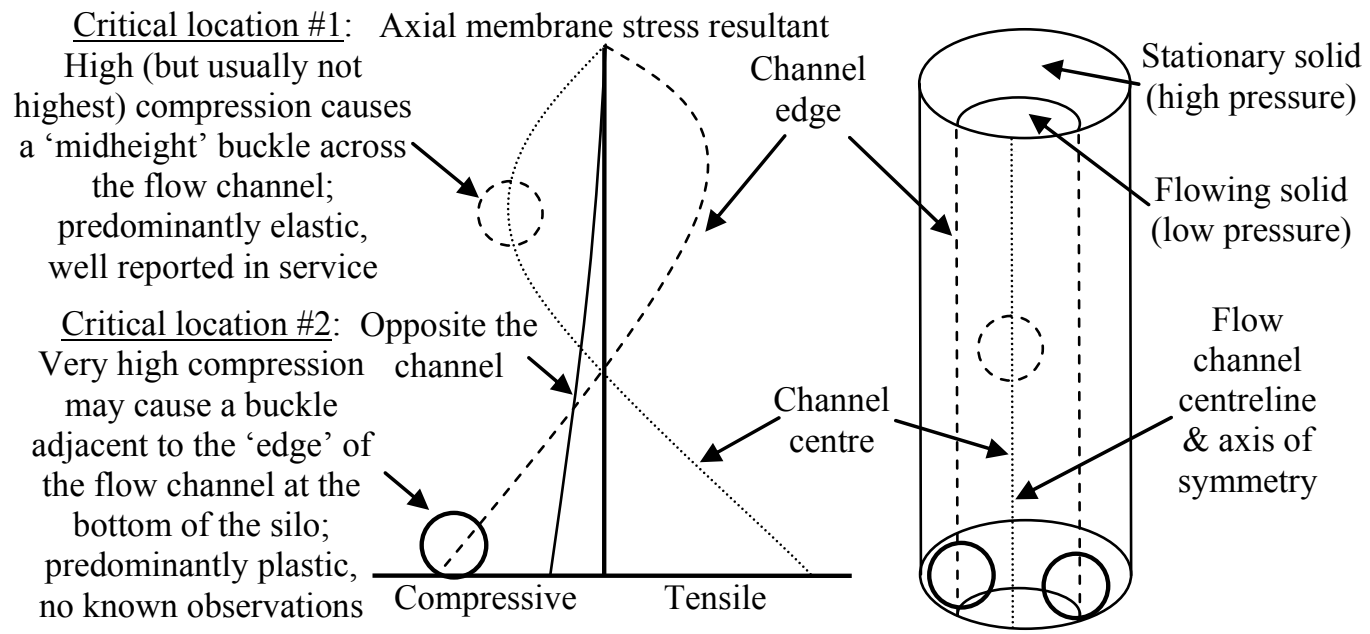

Fig. 3 - Critical locations under eccentric pipe flow in slender silos (after Sadowski \& Rotter, 2011b)

The above studies identified two possible critical locations for localized buckling failure (Fig. 3). The first lies across the flow channel. If the silo wall is of uniform thickness, it is close to the silo midheight, but if the wall has varying thickness it rises slightly to the lowest part of an upper wall strake. This location was first identified by Rotter (1986) as susceptible to the formation of a predominantly elastic buckle due to local high axial compression, and has since become known as the elastic 'midheight' buckling mode. It has been widely observed in practice. The second location lies near the base of the silo near the edge of the flow channel, where an elastic-plastic 'base edge' buckle may potentially form under local high axial compression. There are, however, no known field observations of it, probably because stationary solid exists here inside the silo and restrains the wall against buckling (Rotter \& Zhang, 1990; Knödel et al., 1995).

\subsection{Behavior of the imperfect silo under eccentric pipe flow}

The behavior of imperfect slender metal silos under eccentric pipe flow with the axisymmetric weld depression of Rotter and Teng (1989) was investigated by Sadowski and Rotter (2011b). Though the weld depression is widely considered to be probably the most serious imperfection under uniform axial compression (e.g. Koiter, 1945; 1963; Hutchinson et al., 1971; Rotter, 2004; Song et al., 2004), this imperfection form was found to increase the circumferential bending resistance of the 
Published in: International Journal of Solids and Structures, 50, 781-794.

DOI: http://dx.doi.org/10.1016/j.ijsolstr.2012.11.017

shell, and mostly resulted in an increase in the buckling resistance of the silo under eccentric discharge pressures. The only truly deleterious situations were for impractically small or large channels. Examples of buckling strengths of silos with different imperfection forms are shown in Fig. 4 based on previous work by the authors (Sadowski \& Rotter, 2011b; 2012a). The beneficial effect may be seen to be very large; the axisymmetric weld depression suggests an increase in buckling strength of up to 12 times that of the perfect shell for a mid-sized flow channel. The buckling strength of the imperfect silo has in each case been normalized by the corresponding nonlinear buckling strength of the perfect silo. The silos shown in this figure have a varying wall thickness distribution and were designed according to the hand design provisions of EN 1993-1-6 (2007) and EN 1993-4-1 (2007). The imperfection amplitudes are thus different in each wall strake but they all lie in the range $1 \leq \delta / t \leq 2$, which is consistent with realistic amplitudes for buckling under meridional compression (Rotter, 2004).

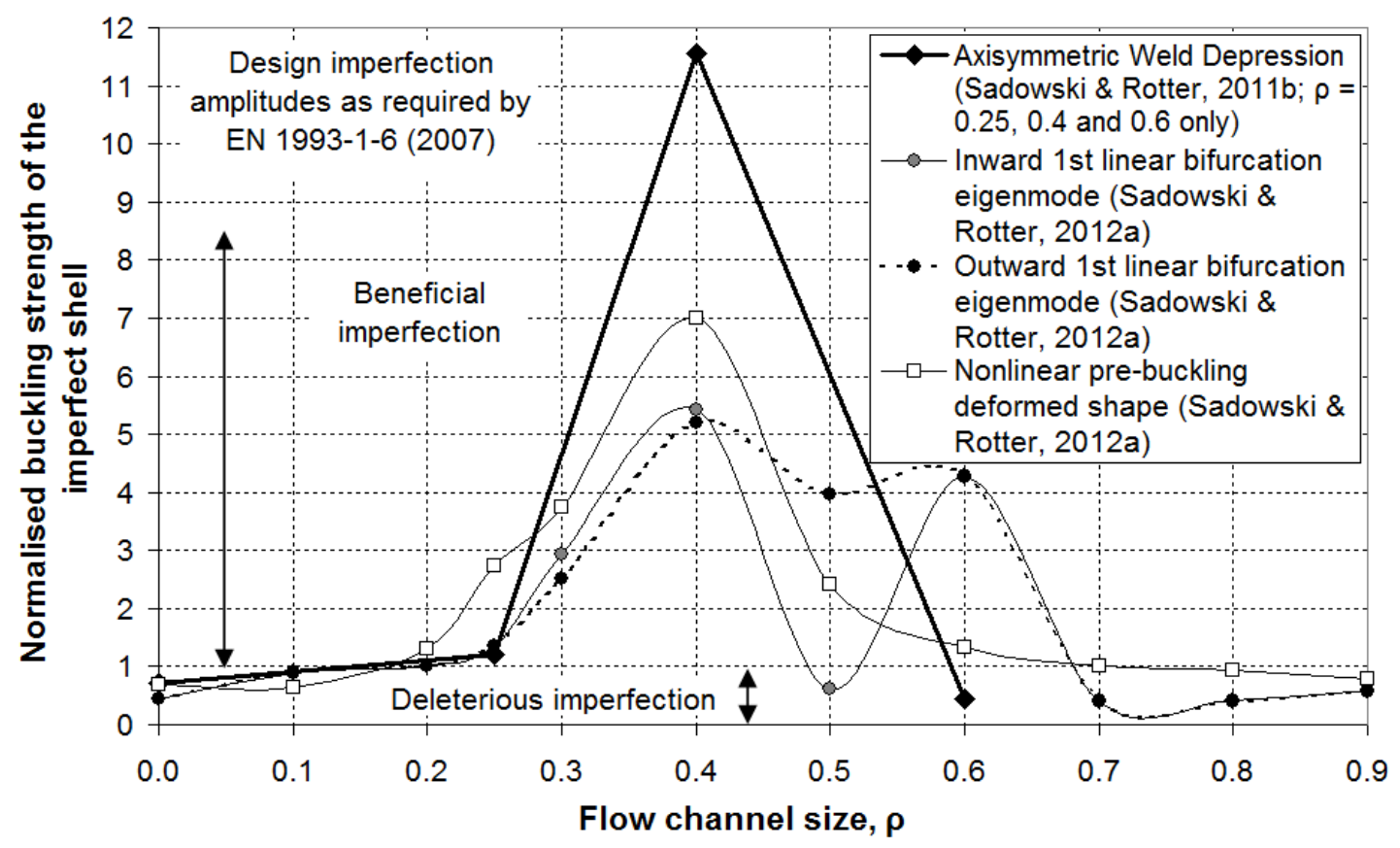

Fig. 4 - Illustration of buckling strengths for silos with different imperfection forms under eccentric discharge (after Sadowski \& Rotter, 2011b and 2012a)

In a second study, Sadowski and Rotter (2012a) investigated the effect of eigenmodeaffine imperfections on a slender silo under eccentric discharge and reached a very similar conclusion for reasons that are explained in detail shortly. These imperfections 
included the first linear bifurcation eigenmode inward, the first linear bifurcation eigenmode outward and the pre-buckling deformed shape from a geometrically nonlinear analysis. Corresponding evaluations (Fig. 4) show that eigenmode-affine imperfection forms are predominantly beneficial under eccentric discharge, but the extent to which they affect the buckling strength is very variable.

The use of the nonlinear pre-buckling deformed shape has been widely proposed as an imperfection form for cylindrical shells (Esslinger \& Geier, 1972; Yamaki, 1984; Guggenberger et al., 2004; Rotter, 2004). The rather interesting reason why this simple choice fails to achieve the desired purpose in this case deserves some explanation. As shown in Fig. 4, the geometrically nonlinear pre-buckling deformations under eccentric discharge were used as an imperfection form by Sadowski \& Rotter (2012a) who found that the pre-buckling deformations near the 'midheight' buckle (Fig. 5c) are substantial and should certainly form part of any adopted imperfection. However, the outcome of imperfect shell buckling calculations did not show them to be significantly deleterious (Fig. 4). Where the silo has a stepped wall with changing thickness, these deformations also include a significant local component arising from local bending at the change of wall thickness above which the 'midheight' buckle forms (Fig. 3). This indentation, due to local axial bending, is amplified by a very local peak in the axial membrane compression, causing approximately axisymmetric waves to develop as the buckling condition is approached, comparable with the development of waves near a boundary (Rotter, 1989). When introduced as an imperfection, these wide but axially short waves lead to an increase in the circumferential bending stiffness of the deformed shell in a manner similar to that of the weld depression (Fig. 8b) (Sadowski \& Rotter, 2011b). The result is a substantial increase in the buckling resistance under eccentric discharge. For this reason, it is not viable to use the nonlinear pre-buckling deformed shape directly as an imperfection form for conditions where stepped wall construction leads to such strengthening. In what follows, the crucial geometric features of the deformed shape (Fig. 6) are isolated and approximated by a novel parametric form, thus omitting the local axial bending associated with a change of plate thickness. 


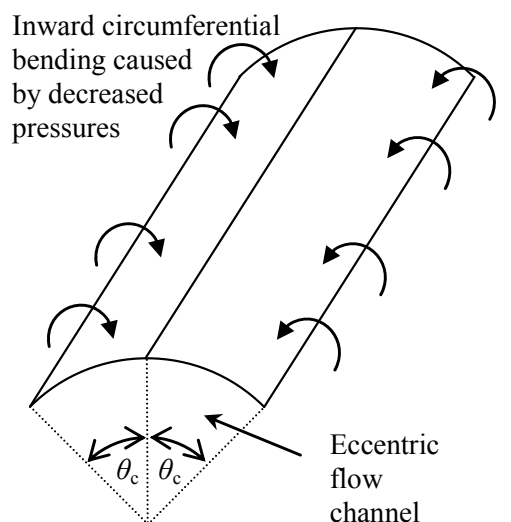

a) Perfect shell

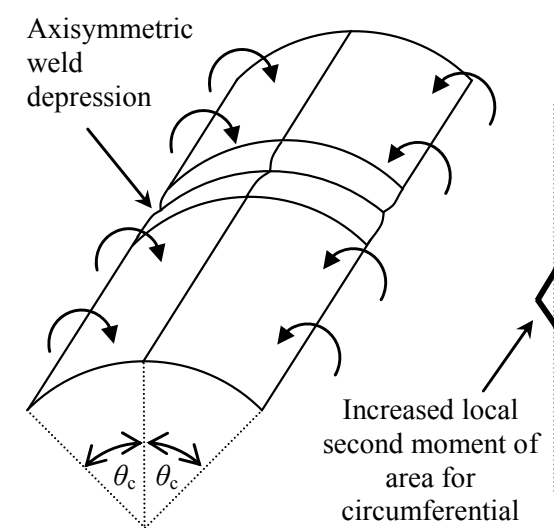
b) Imperfect shell \#1 after Sadowski \& Rotter (2011b)

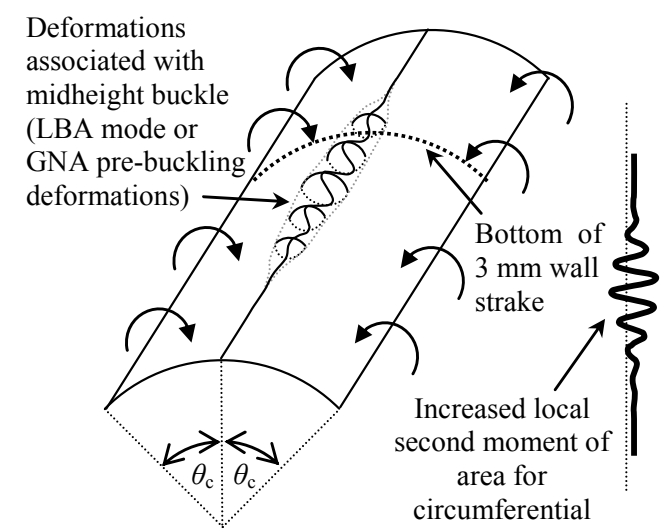

c) Imperfect shell \#2 after Sadowski \& Rotter (2012a)

Fig. 5 - Illustration of the behavior of a slender silo under eccentric discharge with different assumed geometric imperfections

The chief conclusion of all these explorations was that either silos under this load condition are insensitive to imperfections, since it is known that imperfection sensitivity is significantly decreased under highly-localized axial compression (Libai $\&$ Durban, 1977), or that the appropriate deleterious imperfection form had not yet been found.

\subsection{Characteristic features of the pre-buckling deformed shape of the silo}

The pre-buckling deformed shape of a slender silo under eccentric pipe flow is shown in Fig. 6. This deformed shape has been amplified to allow a better appreciation of its characteristic features. The wall of a silo under an axisymmetric normal pressure deforms uniformly radially outward. However, under eccentric pipe flow the normal wall pressure exerted by the flow channel is lower than that exerted by the stationary solid (Figs $1 \& 2$ ), so the outward deformation of the wall near the flow channel is smaller than at other parts of the circumference. This pressure drop thus causes significant local circumferential bending, producing a net inward deformation of the wall at this location. The inward deformation is so great as to cause a significant reduction in the shell curvature, which may be loosely described as local flattening of the wall (Rotter, 1986; Sadowski, 2010; Sadowski \& Rotter, 2012b). Since the shell wall is very stiff for circumferential membrane deformations, the total circumference does not change significantly, and the inward deformation near the flow channel must be accompanied by an outward deformation near the edge of the flow channel. The 
Published in: International Journal of Solids and Structures, 50, 781-794.

DOI: http://dx.doi.org/10.1016/j.ijsolstr.2012.11.017

result is both inward circumferential bending at the flow channel and outward bending at its edges.

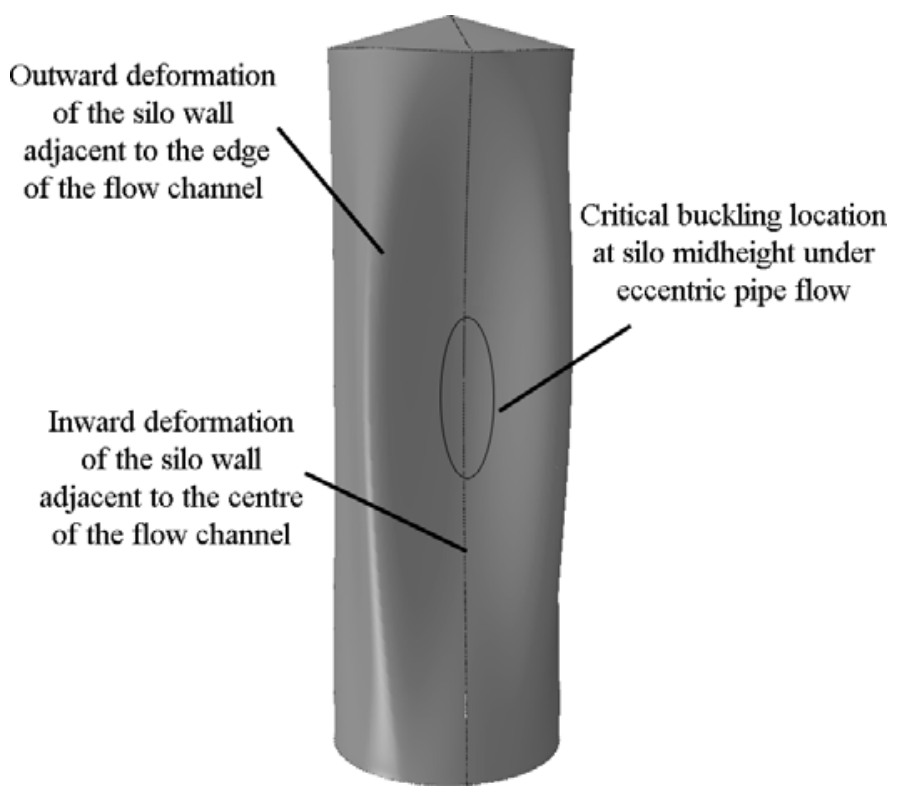

Fig. 6 - Characteristic pre-buckling deformed shape of a slender silo under eccentric pipe flow discharge (Rotter, 1986; Sadowski, 2010; Sadowski \& Rotter, 2012b)

Representative meridional and circumferential forms for the deformed radial shape are characterized in Fig. 7. The meridional form is typically in a single wave throughout the height, though this is not symmetrically distributed with respect to the midheight. The circumferential pattern may be seen to have three distinct components: a large primary inward deformation occurring at the flow channel, a smaller primary outward deformation near the edge of the channel, and an even smaller secondary inward deformation further around the shell circumference. Beyond $90^{\circ}$ on either side of the centre of the flow channel, the shell experiences only negligible deviations from the perfect geometry. 


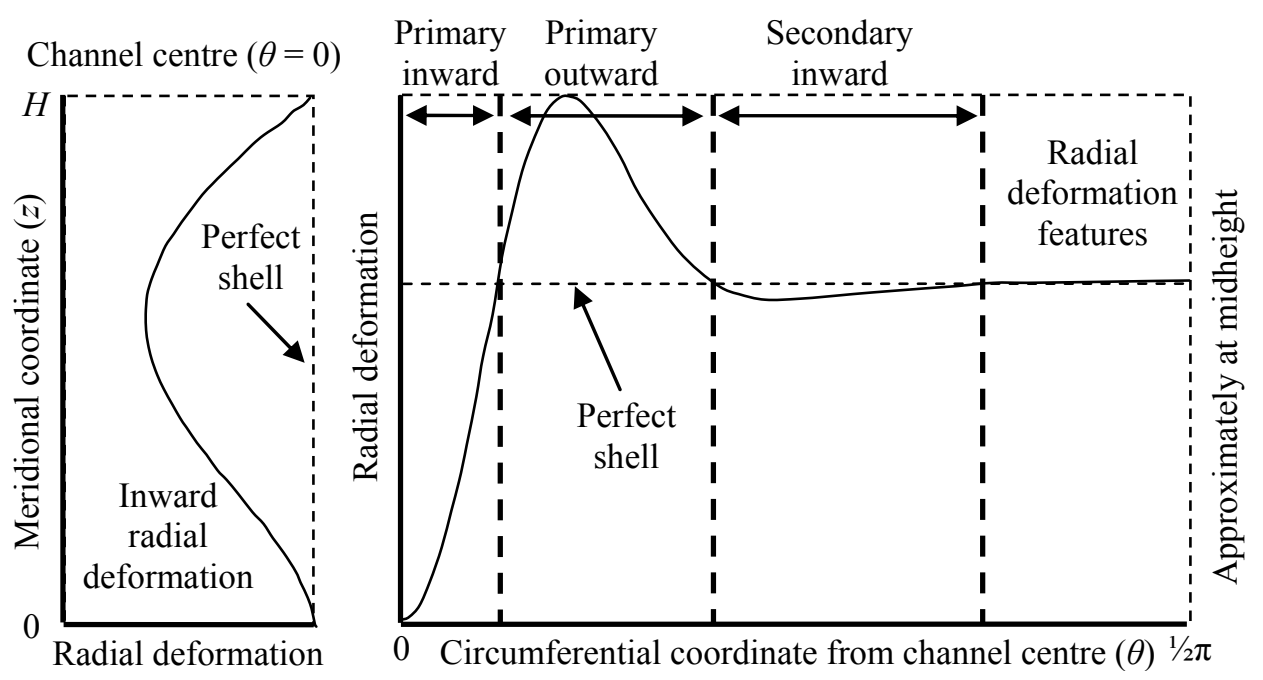

Fig. 7 - Characteristic meridional and circumferential forms of the radial deviation of a slender silo under eccentric pipe flow

It is well understood that the buckling strength of a curved panel is reduced if it becomes flattened and its local effective radius to thickness ratio $R / t$ increases (Rotter, 1985). Further, Sadowski \& Rotter (2012b) showed that this nonlinear flattening phenomenon does indeed have a deleterious effect on the buckling strength of a slender silo under eccentric discharge. A geometric imperfection form which reproduces this feature of the behavior is thus likely to be successful in causing consistent decreases in the predicted buckling strength under eccentric discharge. The imperfection forms that were widely used in the past (e.g. Yamaki, 1984) chiefly failed to achieve this reduction because they do not relate well to the mechanics of the present load case: their basis was often the mechanics of significantly different load conditions (e.g. uniform axial compression).

\section{Introduction to the global imperfection forms used in this study}

In the extensive literature on imperfection sensitivity in shell buckling, the form of an imperfection has traditionally been defined as a local perturbation superimposed on the perfect shell (e.g. Koiter, 1945; 1963; Hutchinson et al, 1971; Yamaki, 1984; Rotter and Teng, 1989). The circumferential and meridional forms of the imperfection 
are usually defined by a relation of the form $\delta=f(z, \theta)$, which is then used to perturb the perfect shell geometry and to generate a local imperfection.

In a break from this tradition, the imperfection forms presented here define the geometry of the complete three-dimensional imperfect shell directly, though still relating the deviation from the perfect shell to a reference imperfection amplitude $\delta_{0}$ at some point in the structure. The global radial coordinate of the imperfect silo wall $r$ is defined here by $r(z, \theta)$ with $\delta(z, \theta)=R-r(z, \theta)$ so that $\delta(z, \theta)=g(z) f(\theta)$, where $g(z)$ and $f(\theta)$ are, respectively, independent meridional and circumferential variations. The range is $z \in[0, H]$ and $\theta \in[0,2 \pi]$, where $H$ is the height of the silo cylinder.

Three different forms of the circumferential component $f(\theta)$ were investigated in this study; these are presented in detail shortly. The meridional component $g(z)$ was in each case assigned a two-part sinusoidal variation consisting of two sine quarterwaves meeting at a chosen height $z_{0}$ (Eq. 1)(Fig. 8). This piecewise continuous meridional form satisfies continuity of both displacement and slope at $z_{0}$, but not of curvature. This was believed to be satisfactory for use as an imperfection form, since $z_{0}$ must usually be chosen close to the silo midheight and the curvature mismatch may thus be small.

$$
g(z)=\left\{\begin{array}{cc}
\sin \left(\frac{\pi}{2} \frac{z}{z_{0}}\right) & \text { for } z \in\left[0, z_{0}\right] \\
\sin \left(\frac{\pi}{2} \frac{(H-z)}{\left(H-z_{0}\right)}\right) & \text { for } z \in\left[z_{0}, H\right]
\end{array}\right.
$$

As illustrated in Fig. 3, the characteristic silo buckling mode associated with eccentric pipe flow is essentially elastic and occurs near silo midheight or at the bottom of a thin upper wall strake. In order to maximize the effectiveness of the imperfection forms proposed in this paper, it is supposed that the base of the strake at which the 'midheight' buckle is predicted to occur from a geometrically nonlinear buckling analysis has been identified, and that this location is chosen to be $z_{0}$ in Eq. 1. If a uniform wall is being analyzed instead, $z_{0}$ is here proposed as the location of the maximum deflection in a propped cantilever (Sadowski and Rotter, 2012b) at $z \approx 0.58 H$ unless there is good reason to do otherwise. In this way, $g(z)$ may be adjusted so that the greatest flattening of the shell occurs close to the location where it 
is likely to be most detrimental, notably near the critical location of the 'midheight' buckle.

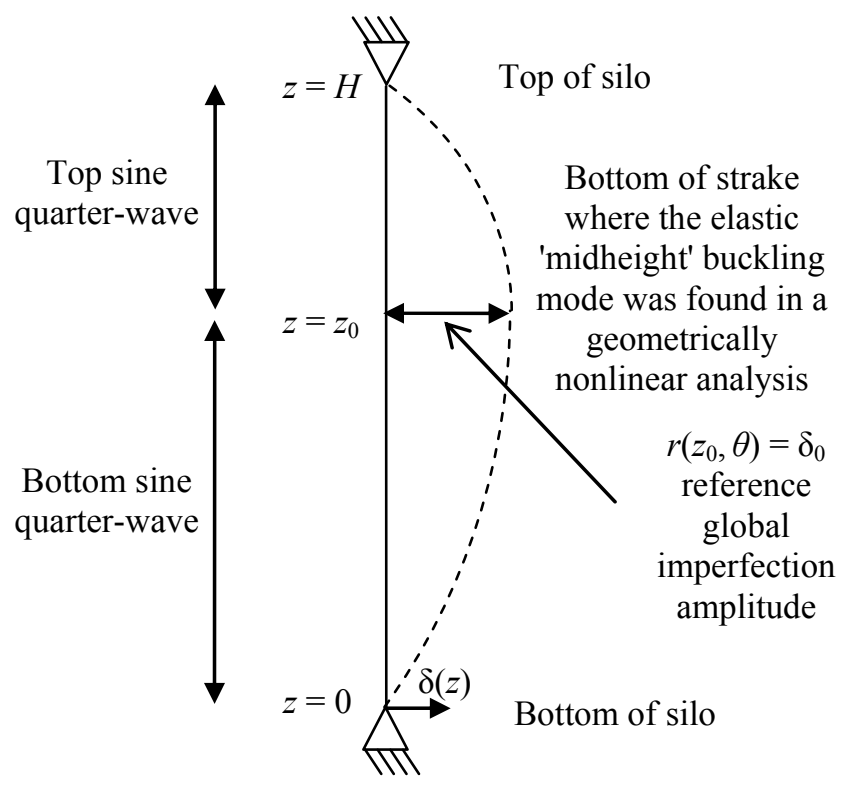

Fig. 8 - Meridional form $g(z)$ of the imperfect shell

\section{Investigation of an imperfection form with local circular flattening}

\subsection{Definition of the shape of the imperfect shell}

A relatively simple geometric representation of local wall flattening was investigated first. An arc of the undeformed circular wall (radius $R$ ) adjacent to the flow channel was replaced with an arc of a circle with a larger radius of curvature $R^{\prime}>R$, thereby making the wall locally flatter up to a local maximum imperfection amplitude $\delta_{0}$ at a height $z_{0}$. This effectively omits both the primary outward and secondary inward radial features seen in Fig. 7, and includes only the primary inward radial feature. The geometry of this imperfection form is shown in local Cartesian coordinates in Fig. 9. A similar imperfection form was used previously in studies of the buckling of spherical caps under external pressure (e.g. Blachut et al., 1990; Blachut and Galletly, 1987; 1990). However, it should be noted that this imperfection form does not contain the same area of shell surface as the perfect shell, so is not altogether likely to be fabricated from pieces of metal that have been cut to the correct size. 


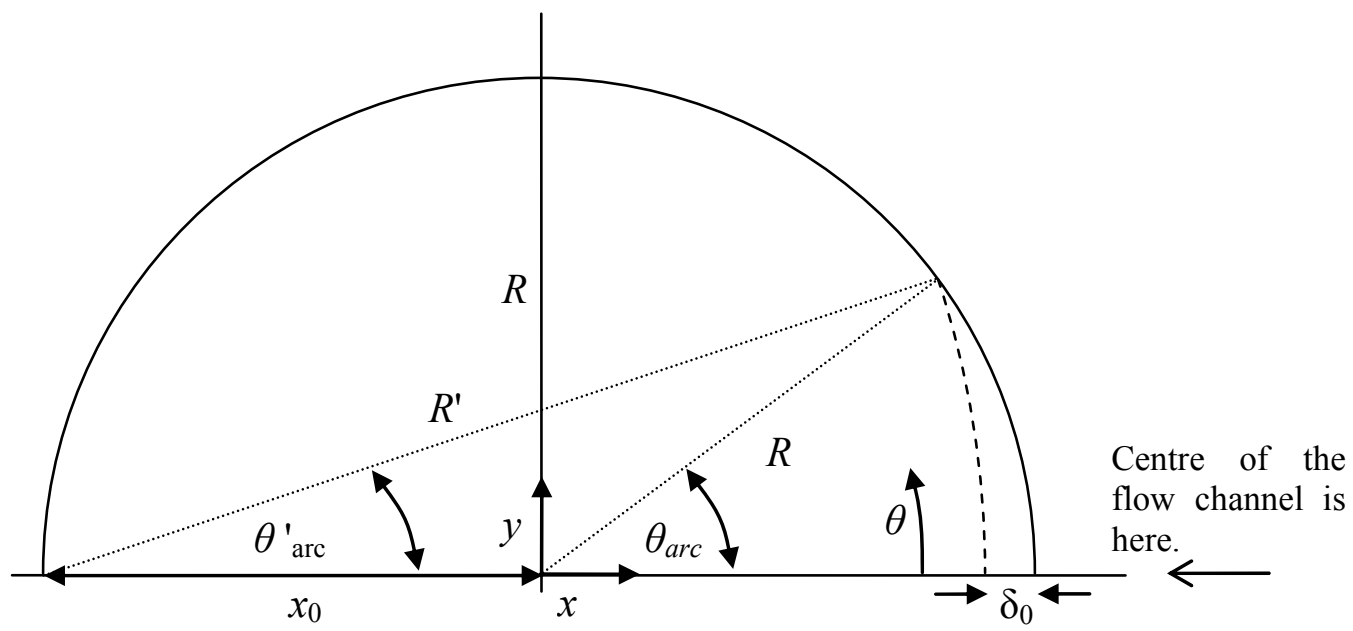

Fig. 9 - Geometry of a local circular flattening imperfection form at height $z_{0}$

The limiting value of $\theta_{\text {arc }}$ may be chosen here, for example, as a multiple of the flow channel wall contact angle $\theta_{\mathrm{c}}$ (Fig. 1). The parameters $x_{0}, R^{\prime}$ and $\theta_{\text {arc }}^{\prime}$ are best computed numerically using the following compatibility relations:

$R \sin \theta_{\text {arc }}=R^{\prime} \sin \theta_{\text {arc }}^{\prime}$

$x_{0}+R \cos \theta_{\text {arc }}=R^{\prime} \cos \theta_{\text {arc }}^{\prime}$

$\delta_{0}=x_{0}+R-R^{\prime}$

If the arc of the flattened circle is to retain the same sense of curvature, the following condition should additionally be satisfied:

$\delta_{0}<R\left(1-\cos \theta_{\text {arc }}\right)$

The coordinates of the circle with the larger radius of curvature are given by:

$x(\theta)=\left(R-\delta_{0}\right) \cos \left(\theta \cdot \frac{\theta_{\text {arc }}^{\prime}}{\theta_{\text {arc }}}\right)-x_{0}$ and $y(\theta)=R \sin \left(\theta \cdot \frac{\theta_{\text {arc }}^{\prime}}{\theta_{\text {arc }}}\right)$

The circumferential component of the radial form becomes:

$f(\theta)=\left\{\begin{array}{cc}R-\sqrt{x(\theta)^{2}+y(\theta)^{2}} & \text { for }-\theta_{\text {arc }} \leq \theta \leq \theta_{\text {arc }} \\ 0 & \text { elsewhere }\end{array}\right.$

The complete imperfect shell is generated by $r(z, \theta)=R-g(z) f(\theta)$, where $g(z)$ and $f(\theta)$ are given by Eqs. 1 and 7 respectively. The flattened arc length $R^{\prime} \times \theta_{\text {arc }}^{\prime}$ is considerably smaller than the undeformed arc length $R \times \theta_{\text {arc }}$, so the imperfect shell 
Published in: International Journal of Solids and Structures, 50, 781-794.

DOI: http://dx.doi.org/10.1016/j.ijsolstr.2012.11.017

has a significantly shorter circumference: as noted above, this is an unsatisfactory feature which may have an unforeseen influence on the behavior.

\subsection{Parametric finite element study of the imperfect shell}

The slender stepped wall thickness silo detailed in Sadowski and Rotter (2012b) was used in this imperfection-sensitivity study. This silo was designed according to the European Standards EN 1993-1-6 (2007) and EN 1993-4-1 (2007) to resist the concentric discharge load case of EN 1991-4 (2006). It has an aspect ratio H/D of 3 $(H=18 \mathrm{~m}, R=3 \mathrm{~m})$, an isotropic steel wall with an elastic modulus of $200 \mathrm{GPa}$, a Poisson's ratio of 0.3 and a thickness distribution varying in a stepwise manner from 3 to $8 \mathrm{~mm}$. The bottom of each strake is critical for buckling under concentric discharge and the design is intentionally insufficient to withstand eccentric discharge pressures of the type considered here. The elastic 'midheight' buckling mode (Fig. 3) was found to occur near the bottom of the thinnest $3 \mathrm{~mm}$ strake, which is at $z_{0}=0.66 \mathrm{H}$ (Fig. 8).

The example silo was analyzed using the finite element software ABAQUS (2009) subject to the eccentric discharge pressures of Rotter (1986) (Fig. 1) assuming a relative flow channel size of $r_{\mathrm{c}}=0.4 R$ and imperfection amplitudes in the range $\delta_{0} \in[0,5 t]$. The spread of the flattened $\operatorname{arc} \theta_{\text {arc }}$ was taken as different multiples of the flow channel wall contact angle $\theta_{\mathrm{c}}=15.1^{\circ}$ (computed according to EN 1991-4), namely $\theta_{\mathrm{arc}}=\theta_{\mathrm{c}}, 2 \theta_{\mathrm{c}}, 4 \theta_{\mathrm{c}}$ and $90^{\circ}$. The resulting imperfection sensitivity curves, normalized by the nonlinear buckling strength (GNA) of the perfect shell, are shown in Fig. 10. This analysis shows that a local increase in the radius of curvature near the flow channel does not lead to a strength-reducing imperfection under eccentric pipe flow discharge. The localized flattened arc instead evidently has a strengthening effect, which declines as the spread of the arc increases. 
Published in: International Journal of Solids and Structures, 50, 781-794.

DOI: http://dx.doi.org/10.1016/j.ijsolstr.2012.11.017

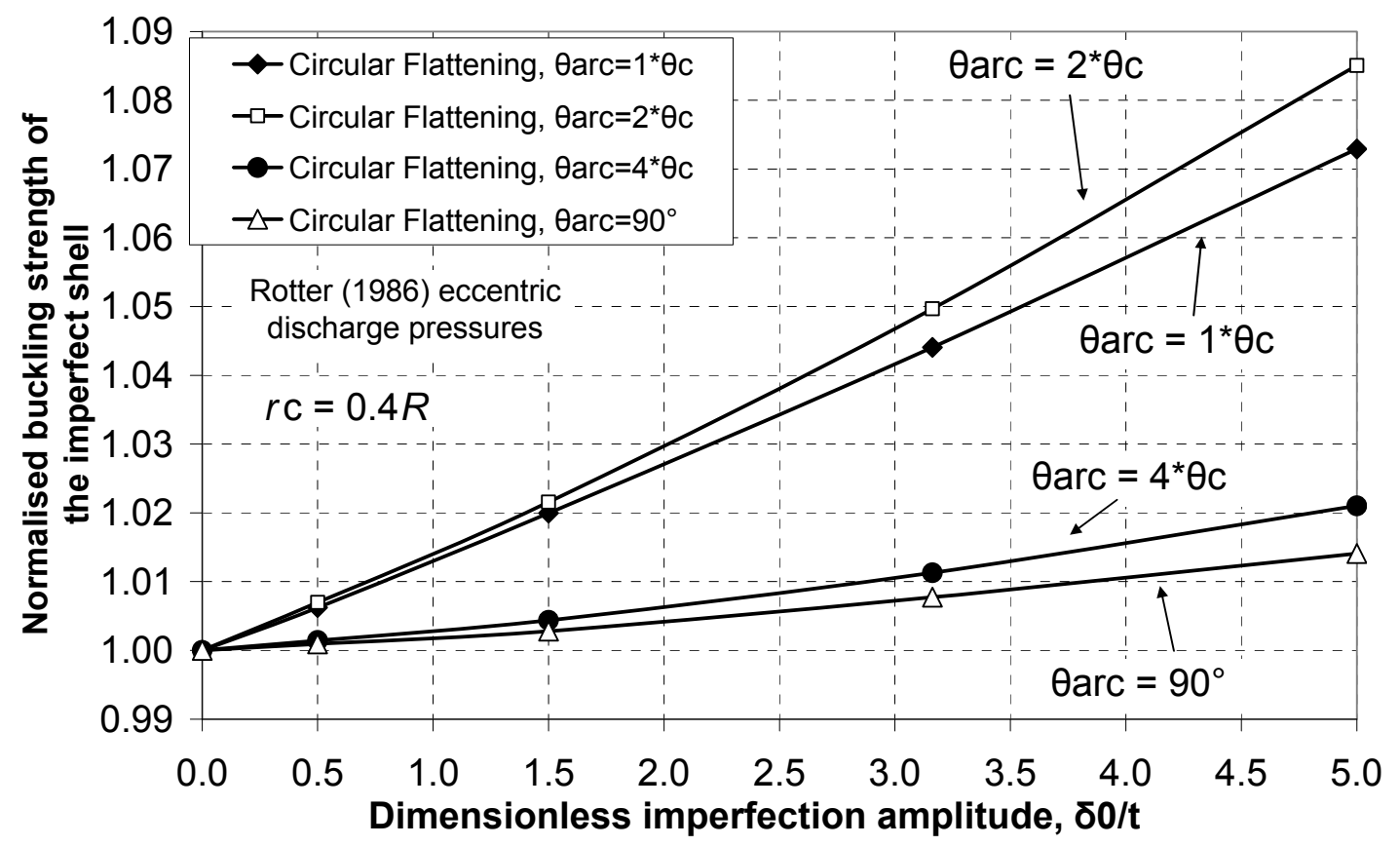

Fig. 10 - Imperfection sensitivity curves for the local circular flattening imperfection form using Rotter (1986) eccentric discharge pressures with $r_{c}=0.4 R$

\section{A novel imperfection form for eccentric pipe flow}

\subsection{Lamé Curves: the superellipse}

It was discovered that the circumferential component of the radial form of the prebuckling deformations near the critical region (Figs 6 \& 7) can be closely approximated by the generalised equation of the Lamé curve (Lamé, 1818; Gridgeman, 1970), also known as the 'superellipse'.

The general formulation of the superellipse in Cartesian coordinates is:

$\left|\frac{x}{a}\right|^{q}+\left|\frac{y}{b}\right|^{p}=1$

This may also be expressed parametrically as:

$x(t)=a \cos ^{2 / q} t$ and $y(t)=b \sin ^{2 / p} t \quad$ with $\quad r(t)=\sqrt{x(t)^{2}+y(t)^{2}}$

where $a, b, p$ and $q$ are positive real numbers and $r(t)$ is the polar form of the circumferential coordinate. An illustration of the great range of different possible shapes which may be obtained using the superellipse is illustrated in Fig. 11. 


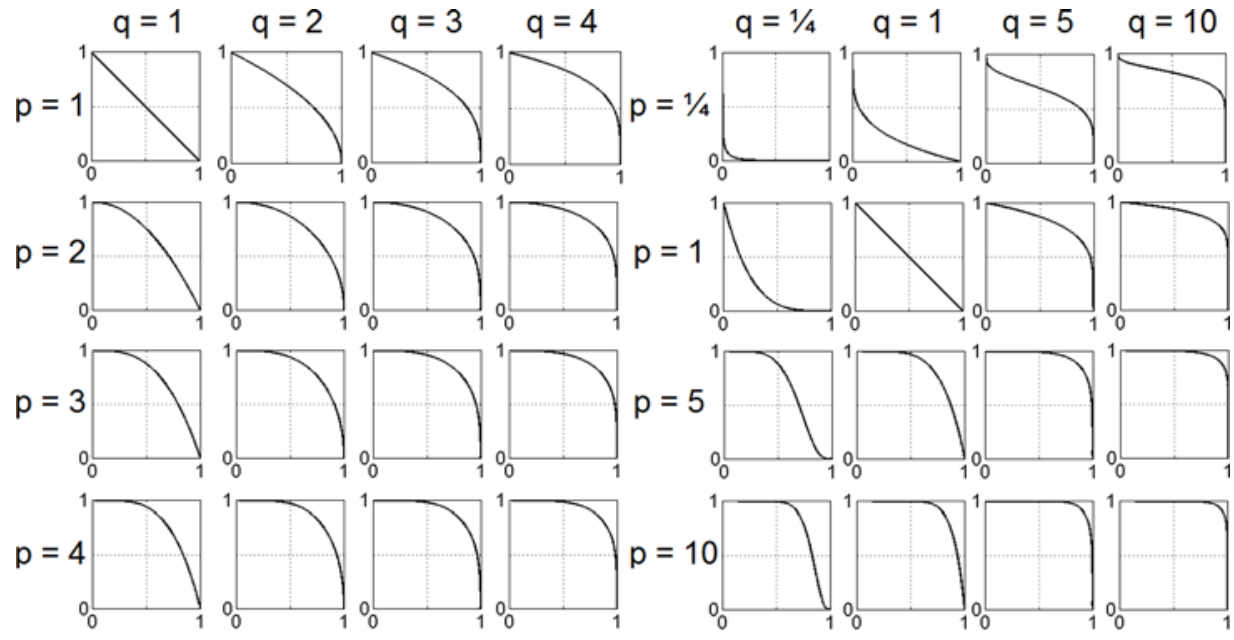

a) Common shapes b) More unusual shapes

Fig. 11 - Superellipses as a function of the parameters $p$ and $q$ (after Sadowski, 2011)

\subsection{First proposed form of the imperfection - Superelliptical flattening with a central inward deviation}

\subsubsection{Circumferential form}

The first proposed shape of the superelliptical imperfection form incorporates each of the deformation features identified in Fig. 7. The wall is allowed to deviate inwards at the flow channel (centered at $\theta=0$ ) up to a reference imperfection amplitude $\delta_{0}$ at the desired level of largest wall flattening $z_{0}$. The wall then deviates outwards to a peak amplitude $n_{\mathrm{a}} \delta_{0}$ at some coordinate $\theta_{\mathrm{a}}$, and then inwards again to an amplitude $n_{\mathrm{b}} \delta_{0}$ at a further coordinate $\theta_{\mathrm{b}}$. The amplitudes of the non-central peak deviations are thus assumed to be related to the central inward deviation by two coefficients $n_{\mathrm{a}}$ and $n_{\mathrm{b}}$ which may be optimized to find the most deleterious flattened shape for buckling. The greatest extent of the region of flattening is $90^{\circ}$ on either side of the centre of the flow channel, beyond which the shell is assumed to be perfect. The geometry is summarized in Fig. 12. 
Published in: International Journal of Solids and Structures, 50, 781-794.

DOI: http://dx.doi.org/10.1016/j.ijsolstr.2012.11.017

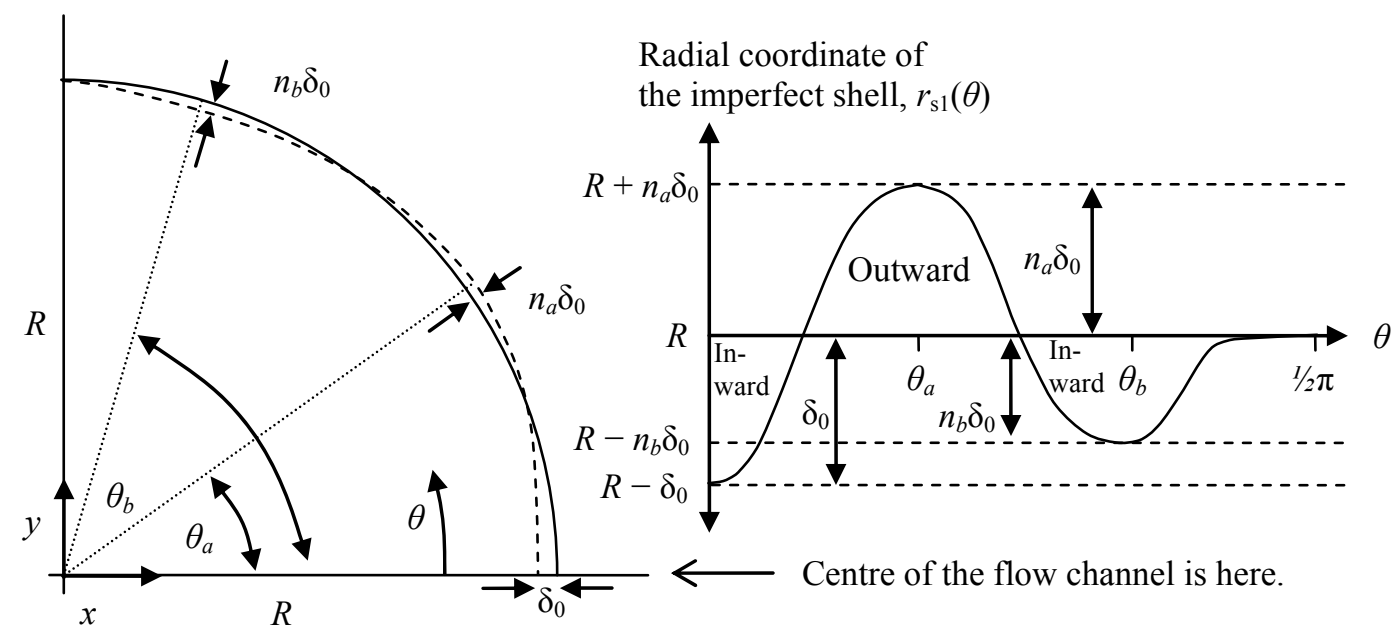

Fig. 12 - Geometry of the first superelliptical imperfection form at height $z_{0}$

It is important to clarify that $\delta_{0}$ is a reference imperfection amplitude for the whole shell, but it need not necessarily define the maximum overall deviation as this depends on the choice of $n_{\mathrm{a}}$ and $n_{\mathrm{b}}$. If either of these is greater than unity, then a larger amplitude of imperfection will occur at the locations $\theta_{\mathrm{a}}$ and/or $\theta_{\mathrm{b}}$. This choice allows full freedom in defining an extensive range of flattened wall forms for the imperfect shell. Lastly, these coefficients should be restricted to $n_{\mathrm{a}} \geq 0$ and $n_{\mathrm{b}} \geq 0$ to prevent a reverse in the sense of curvature of the shell which would lead to significantly different behavior.

The Cartesian coordinates of the imperfect shell are given by:

$$
x_{s 1}(\theta)=\left(R-\delta_{0}\right) \cos ^{2 / q} \theta \text { and } y_{s 1}(\theta)=R \sin ^{2 / p} \theta
$$

The polar forms of the radius and slope of the imperfect shell are thus given by:

$$
\begin{aligned}
r_{s 1}(\theta)=\sqrt{x_{s 1}(\theta)^{2}+y_{s 1}(\theta)^{2}} & =\left(\left(R-\delta_{0}\right)^{2} \cos ^{4 / q} \theta+R^{2} \sin ^{4 / p} \theta\right)^{1 / 2} \\
\frac{d r_{s 1}}{d \theta}(\theta)=2\left(\left(R-\delta_{0}\right)^{2} \cos ^{4 / q} \theta+R^{2} \sin ^{4 / p} \theta\right)^{-1 / 2} \times & {\left[\frac{R^{2}}{p} \cot \theta \sin ^{4 / p} \theta-\frac{\left(R-\delta_{0}\right)^{2}}{q} \tan \theta \cos ^{4 / q} \theta\right] }
\end{aligned}
$$

The circumferential component of the radial form of becomes: 
Published in: International Journal of Solids and Structures, 50, 781-794.

DOI: http://dx.doi.org/10.1016/j.ijsolstr.2012.11.017

$$
f_{s 1}(\theta)=\left\{\begin{array}{cc}
R-r_{s 1}(\theta) & \text { for }-\frac{\pi}{2} \leq \theta \leq \frac{\pi}{2} \\
0 & \text { elsewhere }
\end{array}\right.
$$

The complete imperfect shell is thus generated by $r(z, \theta)=R-g(z) f_{\mathrm{s} 1}(\theta)$, where $g(z)$ and $f_{\mathrm{s} 1}(\theta)$ are given by Eqs. 1 and 13 respectively. The parameters $p, q, \theta_{\mathrm{a}}$ and $\theta_{\mathrm{b}}$ may be found numerically by satisfying the four boundary conditions:

$r_{s 1}\left(\theta_{a}\right)=R+n_{a} \delta ; \quad r_{s 1}\left(\theta_{b}\right)=R-n_{b} \delta ; \quad \frac{d r_{s 1}}{d \theta}\left(\theta_{a}\right)=\frac{d r_{s 1}}{d \theta}\left(\theta_{b}\right)=0 ;$

If $\delta_{0}=0$ and $p=q=2$, then Eq. 13 naturally generates a perfect shell. A flattened shape with geometric features similar to those in Fig. 7 is obtained when $p>2, q<2$ and $\delta_{0}>0$. The variation of the powers $p$ and $q$ with $\delta_{0} / t$ for a typical thin shell with $R / t=1000$ is shown in Fig. 13 for combinations of $n_{\mathrm{a}}$ and $n_{\mathrm{b}}$ equal to 0.1 or 1 (i.e. an order of magnitude apart). It appears that the greater the relative difference between $n_{\mathrm{a}}$ and $n_{\mathrm{b}}$, the greater the change in both $p$ and $q$ from the reference value of 2 . Surprisingly, $p$ and $q$ follow an approximately linear relation with $\delta_{0} / t$ which may allow them to be obtained easily using a simple empirical equation rather than the above numerical procedure.

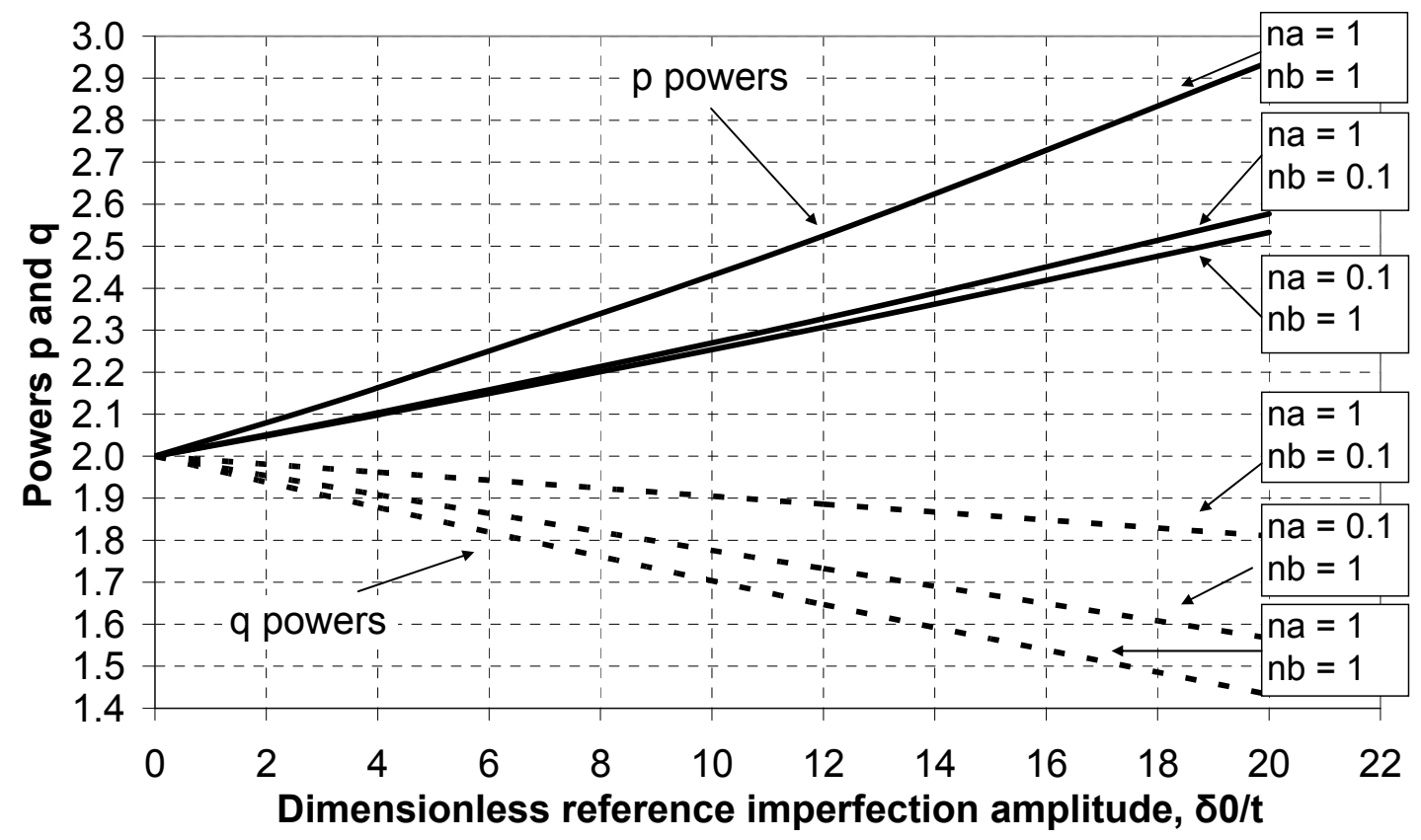

Fig. 13 - Typical variation of powers $p$ and $q$ with $\delta_{0} / t$ for the first superelliptical imperfection form $(R / t=1000)$ 
Published in: International Journal of Solids and Structures, 50, 781-794.

DOI: http://dx.doi.org/10.1016/j.ijsolstr.2012.11.017

An important consideration is the change in the perimeter of the imperfect shell with $\delta_{0} / t$. The length of the total circumference is an aspect that is usually strictly controlled during silo construction, and even if the quality of the welding of wall strakes is poor, the sum of their lengths is effectively fixed. The arc length of the imperfect portion of the shell may be obtained by numerical integration of the following:

$s_{s 1}=\int_{0}^{\frac{\pi}{2}} \sqrt{\left(\frac{d x_{s 1}}{d \theta}\right)^{2}+\left(\frac{d y_{s 1}}{d \theta}\right)^{2}} d \theta$

The variation of the imperfect perimeter normalized by the perfect perimeter $(2 \pi R)$ is shown in Fig. 14 as a function of $\delta_{0} / t$ for $n_{\mathrm{a}}$ and $n_{\mathrm{b}}$ equal to 0.1 and 1 , as above. The change in the full circumference is of the order of 1 to $2 \%$ for this range of $\delta_{0} / t$, which is arguably possible in practice. In the parametric studies presented later, it was found that the change in the circumference did not exceed $5 \%$.

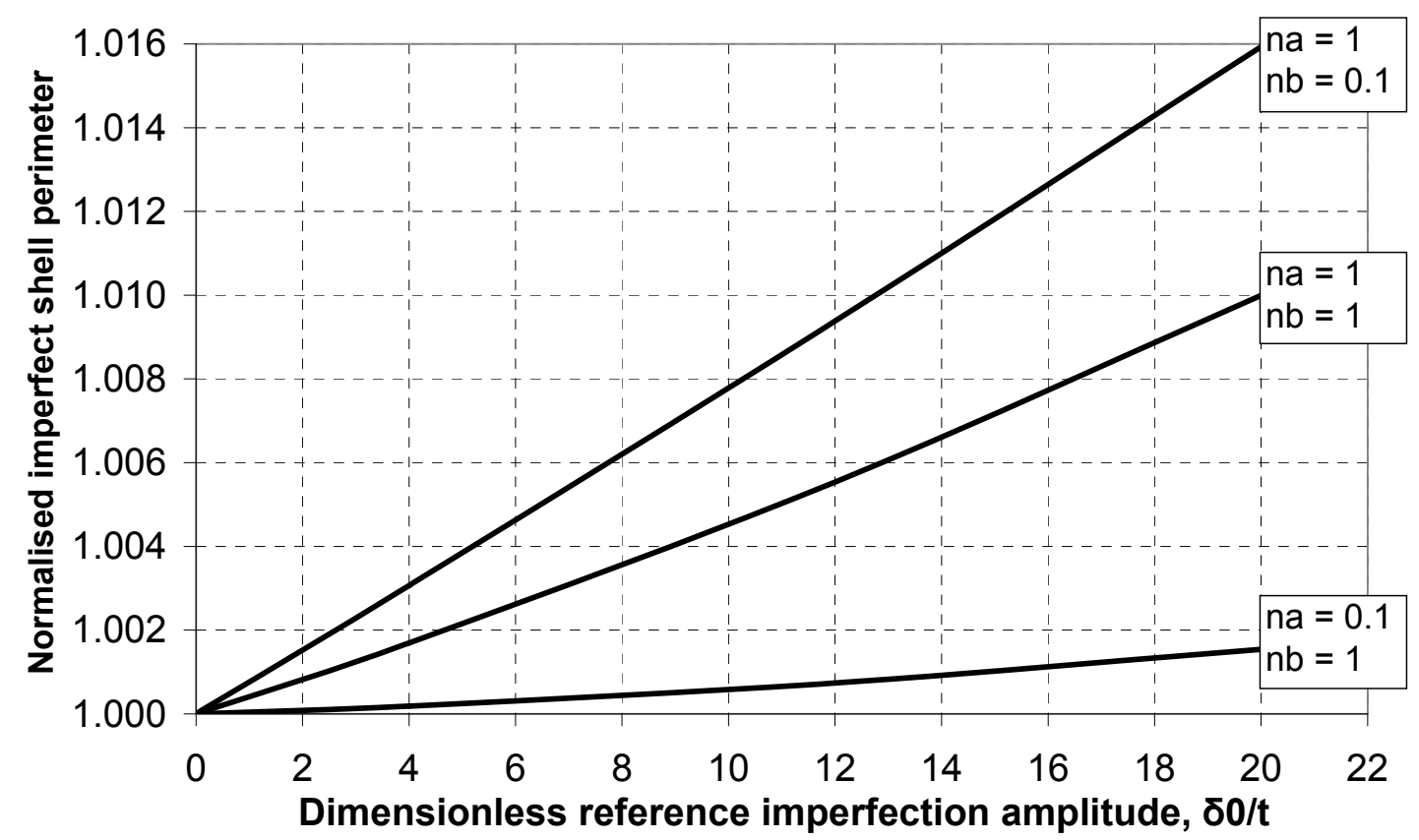

Fig. 14 - Typical variation of the normalized imperfect perimeter with $\delta_{0} / t$ for the first superelliptical imperfection form $(R / t=1000)$ 
Published in: International Journal of Solids and Structures, 50, 781-794.

DOI: http://dx.doi.org/10.1016/j.ijsolstr.2012.11.017

\subsubsection{Imperfection sensitivity study of the first superelliptical imperfection form under the Rotter (1986) eccentric discharge pressures}

A set of geometrically nonlinear buckling analyses was carried out on the slender example silo introduced earlier. This silo was modeled in ABAQUS (2009) as an imperfect shell with the first superelliptical imperfection form subject to the eccentric discharge pressure model of Rotter (1986), assuming a medium-sized flow channel $r_{\mathrm{c}}=0.4 R$ and reference imperfection amplitudes in the range $\delta_{0} \in[0,15 t]$. The following four combinations of $n_{\mathrm{a}}$ and $n_{\mathrm{b}}$ were considered: Form A $\left(n_{\mathrm{a}}, n_{\mathrm{b}}\right)=(1,1)$ where all deviations are equal, Form $\mathrm{B}\left(n_{\mathrm{a}}, n_{\mathrm{b}}\right)=(2,1)$ where the frontal flattening is more pronounced, Form $\mathrm{C}\left(n_{\mathrm{a}}, n_{\mathrm{b}}\right)=(2,1 / 4)$ where the inward deviation away from the centre of the channel is made very small and Form $\mathrm{D}\left(n_{\mathrm{a}}, n_{\mathrm{b}}\right)=(4,1)$ where the frontal flattening is even more pronounced. Normalized imperfection sensitivity curves for these four forms are shown in Fig. 15. The normalization for this and each subsequent figure is always with respect to the nonlinear buckling strength (GNA) of the perfect shell.

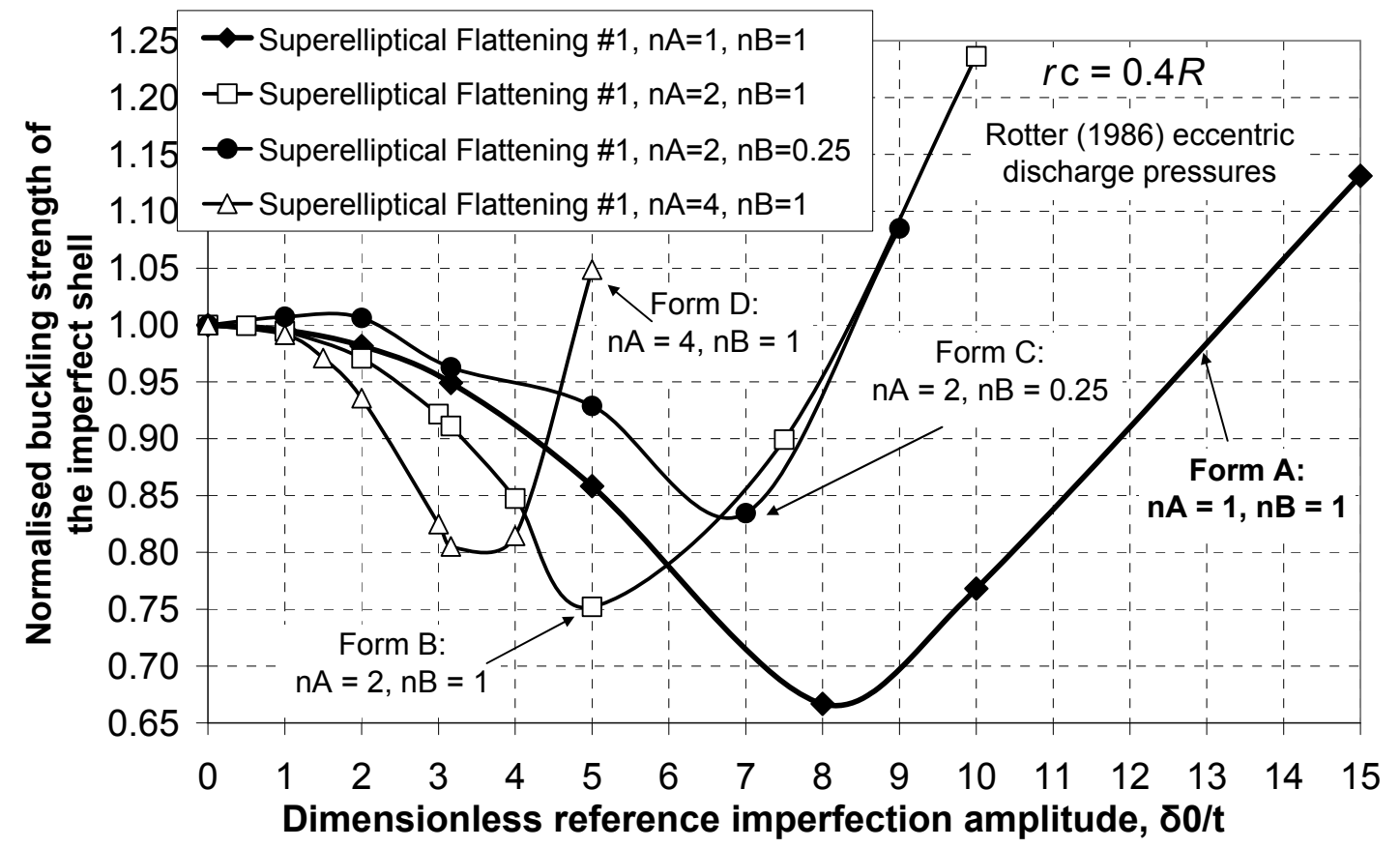

Fig. 15 - Imperfection sensitivity curves for the first superelliptical imperfection form under the Rotter (1986) eccentric discharge pressures with $r_{\mathrm{c}}=0.4 R$

The curve for Form A $\left(n_{\mathrm{a}}, n_{\mathrm{b}}\right)=(1,1)$ suggests that this imperfection form is indeed significantly detrimental to the buckling strength of the silo up to a certain amplitude. 
Oddly, as $\delta_{0}$ increases beyond $8 t$ the curve suddenly turns and the imperfection produces a beneficial effect after 13t. It is not yet clear why this abrupt reversal occurs. When the frontal flattening (primary inward radial feature, Figs 7 and 12) is made more pronounced by increasing the value of $n_{\mathrm{a}}$, an increasingly deleterious effect is obtained at smaller values of $\delta_{0} / t$. For example, for Form B $\left(n_{\mathrm{a}}=2\right)$ the decrease in buckling strength is $7.5 \%$ at $\delta_{0}=3 t$, but the decrease rises to $20 \%$ for Form $\mathrm{D}\left(n_{\mathrm{a}}=4\right)$. However, although these higher values of $n_{\mathrm{a}}$ result in more significant buckling strength losses at small amplitudes $\delta_{0} / t$, they also produce a sudden turn at lower amplitudes $\delta_{0} / t$ values and the subsequent rise is steeper. Indeed, for $n_{\mathrm{a}}=4$ and $\delta_{0} \geq 8 t$ the strength gains become enormous. Lastly, the secondary inward radial feature should not be neglected by making $n_{\mathrm{b}}$ too small, as it may be seen in Form $\mathrm{C}\left[\left(n_{\mathrm{a}}, n_{\mathrm{b}}\right)=(2,1 / 4)\right]$ where smaller reductions in the buckling strength are seen at lower amplitudes than for Form B $\left[\left(n_{\mathrm{a}}, n_{\mathrm{b}}\right)=(2,1)\right]$.

\subsubsection{Imperfection sensitivity study of the first superelliptical imperfection form under the EN 1991-4 (2006) codified eccentric discharge pressures}

It is now evident that local flattening of the silo wall adjacent to the flow channel may be a suitable imperfection form for one form of eccentric discharge pressures (Fig. 1). The next exploration sought to determine whether this imperfection form may also be suitable when slight changes are made to the circumferential pressure distribution, as in the codified eccentric discharge pressure distribution of EN 1991-4 (2006), which includes a large increased normal pressure patch just outside the flow channel and is arguably more severe on the structure than that of Rotter (1986). The resulting imperfection sensitivity curves for the three flow channel sizes recommended in EN $1991-4, r_{\mathrm{c}}=0.25 R, 0.4 R$ and $0.6 R$, are summarized in Fig. 16 assuming reference imperfection amplitudes $\delta_{0} \in[0,5 t]$ and the flattened shape coefficients of Form D $\left(n_{\mathrm{a}}, n_{\mathrm{b}}\right)=(4,1)$. 
Published in: International Journal of Solids and Structures, 50, 781-794.

DOI: http://dx.doi.org/10.1016/j.ijsolstr.2012.11.017

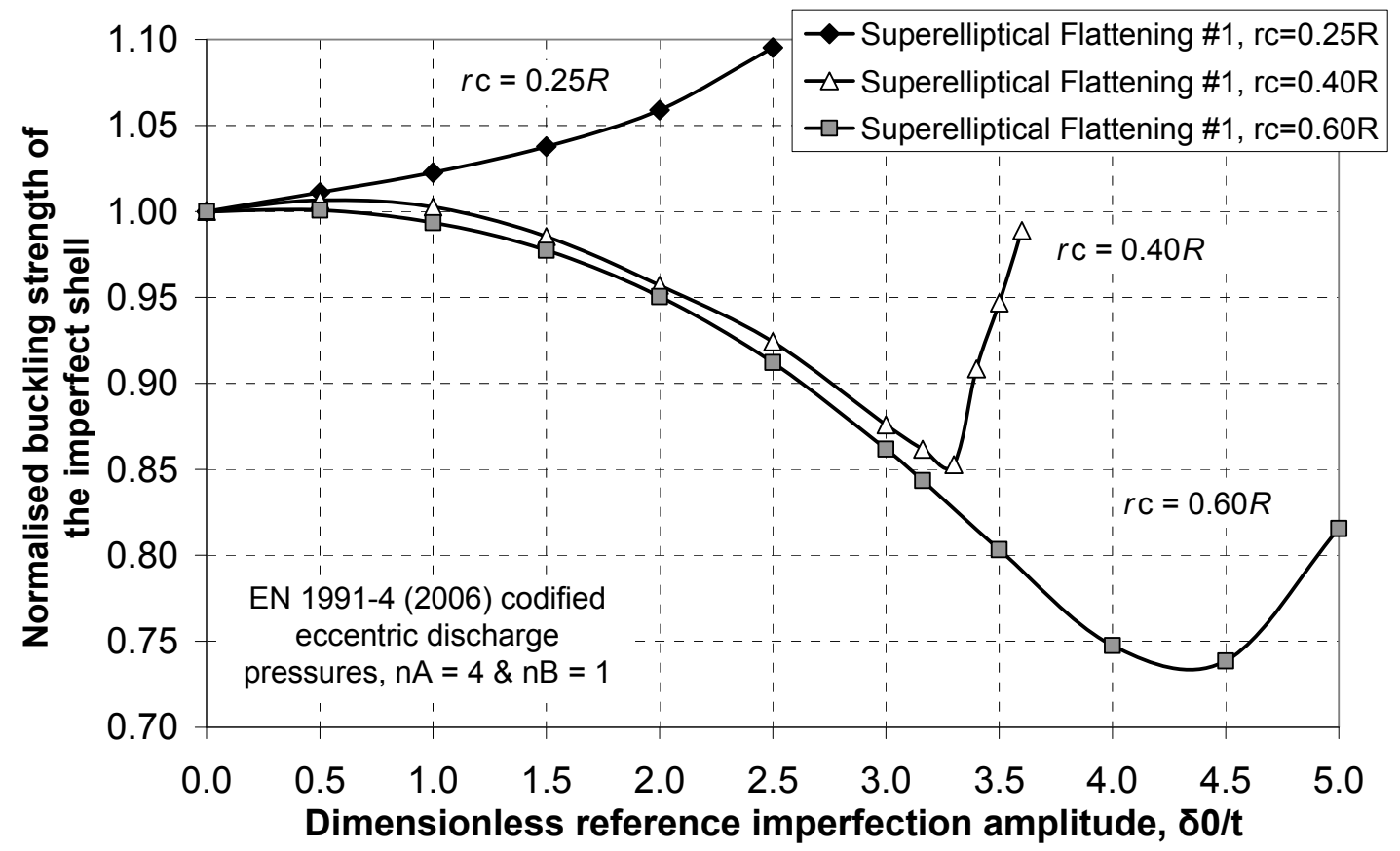

Fig. 16 - Imperfection sensitivity curves for the first superelliptical imperfection form under the EN 1991-4 (2006) codified eccentric discharge pressures

The imperfection sensitivity curves in Fig. 16 suggest that this superelliptical flattening imperfection form may be similarly deleterious to the buckling strength of a silo under this alternative and more punishing formulation of eccentric discharge pressures. For $r_{\mathrm{c}}=0.4 R$ and $0.6 R$, this imperfection form is predicted to lead to nontrivial reductions in the buckling strength for values of $\delta_{0} / t$ up to about 3.2 and 4.4 respectively. However, beyond these amplitudes there is once again a sudden and steep reduction in imperfection sensitivity, as in Fig. 15.

For the smallest channel size of $r_{\mathrm{c}}=0.25 R$, this imperfection is predicted to become beneficial at all amplitudes. In a previous study, Sadowski \& Rotter (2011b) found that the characteristic slender silo structural behavior under EN 1991-4 eccentric discharge pressures (Fig. 3) does not develop when the flow channel is smaller than about $r_{\mathrm{c}}=0.3 R$. Crucially, the deformed shape of the silo wall under the action of a small flow channel does not resemble the characteristic shape illustrated Figs 6 and 7 and thus the present superelliptical flattening form has no physical basis to act as an effective imperfection. 


\subsection{Second proposed form of the imperfection - Superelliptical flattening with no central inward deviation}

\subsubsection{Circumferential form}

The second proposed shape for a superelliptical imperfection incorporated only the primary outward and secondary inward radial features identified in Fig. 7. It is assumed that there is no inward deviation of the shell wall at the centre of the flow channel at $\theta=0$. This choice was made in order to obtain a broader picture of the effect of different flattened shapes on the behavior of imperfect slender silos under eccentric discharge. The flattened shape of the wall thus consists of an outward deviation at $\theta_{\mathrm{a}}>0$ near the edge of the flow channel to an amplitude $\delta_{0}$ and an inward deviation of the shell further around the circumference to an amplitude $m \delta_{0}$ at $\theta_{\mathrm{b}}>\theta_{\mathrm{a}}$. The amplitude of the inward feature is assumed to be related to the reference amplitude $\delta_{0}$ by a coefficient $m$ which controls the extent of the flattening. The previous discussion on the possible choices of $n_{\mathrm{a}}$ and $n_{\mathrm{b}}$ for the first superelliptical form may be extended to $m$. The geometry is summarized in Fig. 17.

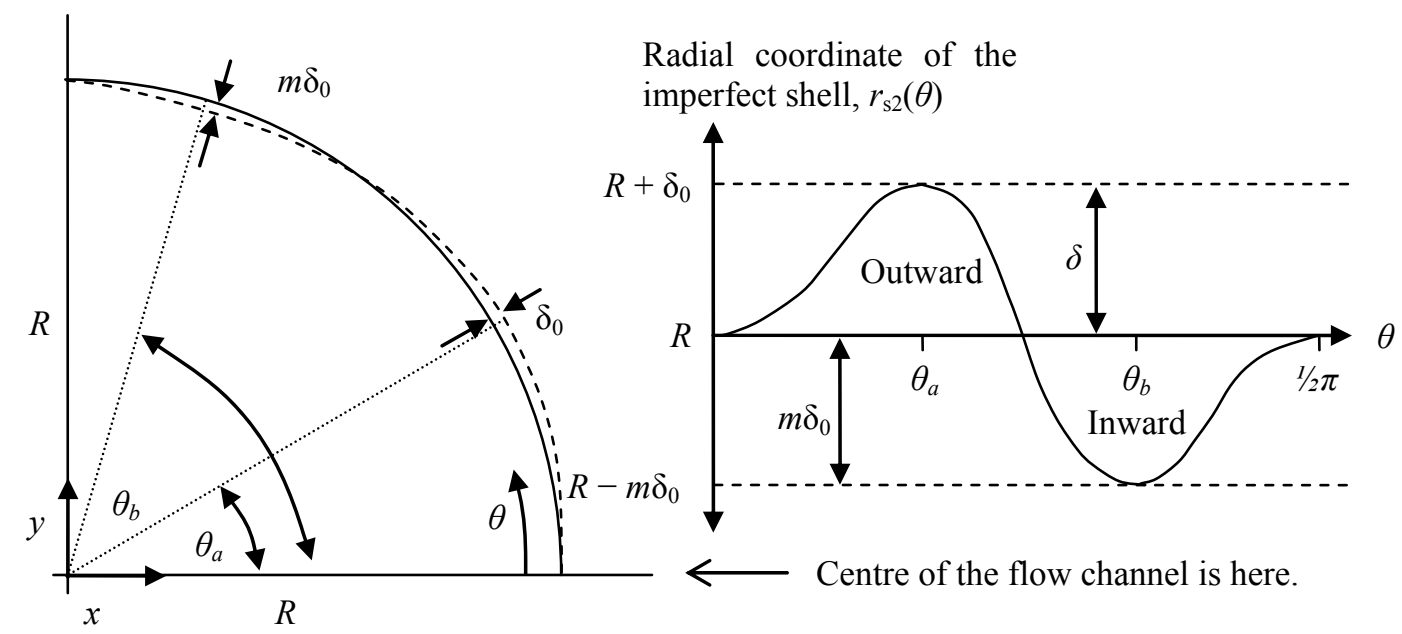

Fig. 17 - Geometry of the second superelliptical imperfection form at height $z_{0}$

The Cartesian coordinates of the imperfect shell are given by:

$x_{s 2}=R \cos ^{2 / q} \theta$ and $y_{s 2}=R \sin ^{2 / p} \theta$

The polar forms of the radius and slope of the imperfection shell are:

$r_{s 2}(\theta)=\sqrt{x_{s 2}(\theta)^{2}+y_{s 2}(\theta)^{2}}=R\left(\cos ^{4 / q} \theta+\sin ^{4 / p} \theta\right)^{1 / 2}$ 


$$
\frac{d r_{s 2}}{d \theta}(\theta)=2 R\left(\cos ^{4 / q} \theta+\sin ^{4 / p} \theta\right)^{-1 / 2}\left[\frac{1}{p} \cot \theta \sin ^{4 / p} \theta-\frac{1}{q} \tan \theta \cos ^{4 / q} \theta\right]
$$

The circumferential component of the radial form of this imperfection is thus:

$$
f_{s 2}(\theta)=\left\{\begin{array}{cc}
R-r_{s 2}(\theta) & \text { for }-\frac{\pi}{2} \leq \theta \leq \frac{\pi}{2} \\
0 & \text { elsewhere }
\end{array}\right.
$$

As before, the complete imperfect shell may be generated by $r(z, \theta)=R-g(z) f_{\mathrm{s} 2}(\theta)$, where $g(z)$ and $f_{\mathrm{s} 2}(\theta)$ are given by Eqs 1 and 19 respectively. The parameters $p, q, \theta_{\mathrm{a}}$ and $\theta_{\mathrm{b}}$ may similarly be found numerically to satisfy the four boundary conditions:

$$
r_{s 2}\left(\theta_{a}\right)=R+\delta ; \quad r_{s 2}\left(\theta_{b}\right)=R-m \delta ; \quad \frac{d r_{s 2}}{d \theta}\left(\theta_{a}\right)=\frac{d r_{s 2}}{d \theta}\left(\theta_{b}\right)=0
$$

The desired flattened shape is obtained when $p>2, q<2$ and $\delta_{0}>0$, with $\delta_{0}=0$ and $p=q=2$ generating a perfect shell. The variation of the powers $p$ and $q$ with $\delta_{0} / t$ follows a nearly linear relationship similar to that for the first superelliptical imperfection form (Fig. 13). The imperfect perimeter length may similarly be calculated using Eq. 16, with subscripts $s 2$ instead of $s 1$. This evaluation suggests a length change below $1 \%$, varying almost linearly with both $m$ and $\delta_{0} / t$ (cf. Fig. 13).

\subsubsection{Imperfection sensitivity study of the second superelliptical imperfection form under the Rotter (1986) eccentric discharge pressures}

The effect of this imperfection form on the buckling strength of the example slender silo was explored next. A series of nonlinear elastic buckling analyses were again performed on this silo with ABAQUS (2009) assuming the eccentric discharge pressures of Rotter (1986) with $r_{\mathrm{c}}=0.25 R, 0.4 R$ and $0.6 R$. Imperfection amplitudes were taken in the range $\delta_{0} \in[0,10 t]$ for $r_{\mathrm{c}}=0.25 R$ and $\delta_{0} \in[0,20 t]$ for the larger flow channels. These values certainly cause rather large deviations of the wall from that of a perfect shell, but such high deviations can occur over the longer perimeter lengths seen here, in accordance with the different imperfection measuring system commonly used (EN 1993-1-6, 2007). Values for the coefficient $m$ were taken as 0.5, 1 and 2 to determine the effect of varying the degree of wall flattening. The resulting imperfection sensitivity curves are shown in Figs 18 to 20. 
Published in: International Journal of Solids and Structures, 50, 781-794.

DOI: http://dx.doi.org/10.1016/j.ijsolstr.2012.11.017

This imperfection form causes consistent decreases in the predicted nonlinear buckling strength for all three flow channel sizes for amplitudes up to at least $\delta_{0} / t=10$. Beyond a certain value of $\delta_{0} / t$, the buckling strength rises again in a similar manner to the first formulation (Fig. 15). The amplitude $\delta_{0} / t$ at which the worst effect occurs reduces as the coefficient $m$ is increased, but the subsequent rise is less steep and abrupt. A value of $m \geq 1$ is therefore suitable for studies of this imperfection form.

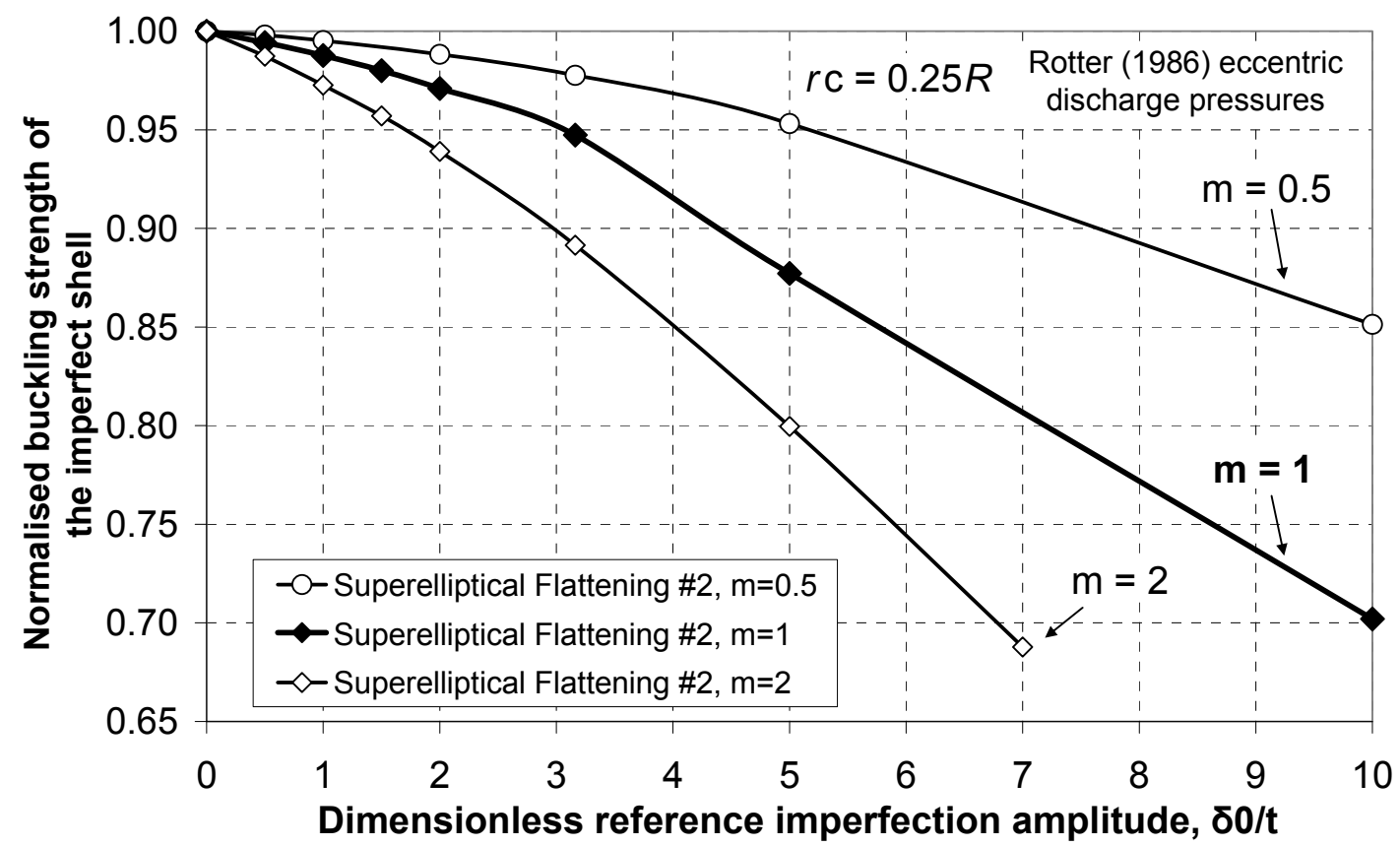

Fig. 18 - Imperfection sensitivity curves for the second superelliptical imperfection form under the Rotter (1986) eccentric discharge pressures with $r_{\mathrm{c}}=0.25 R$ 
Published in: International Journal of Solids and Structures, 50, 781-794.

DOI: http://dx.doi.org/10.1016/j.ijsolstr.2012.11.017

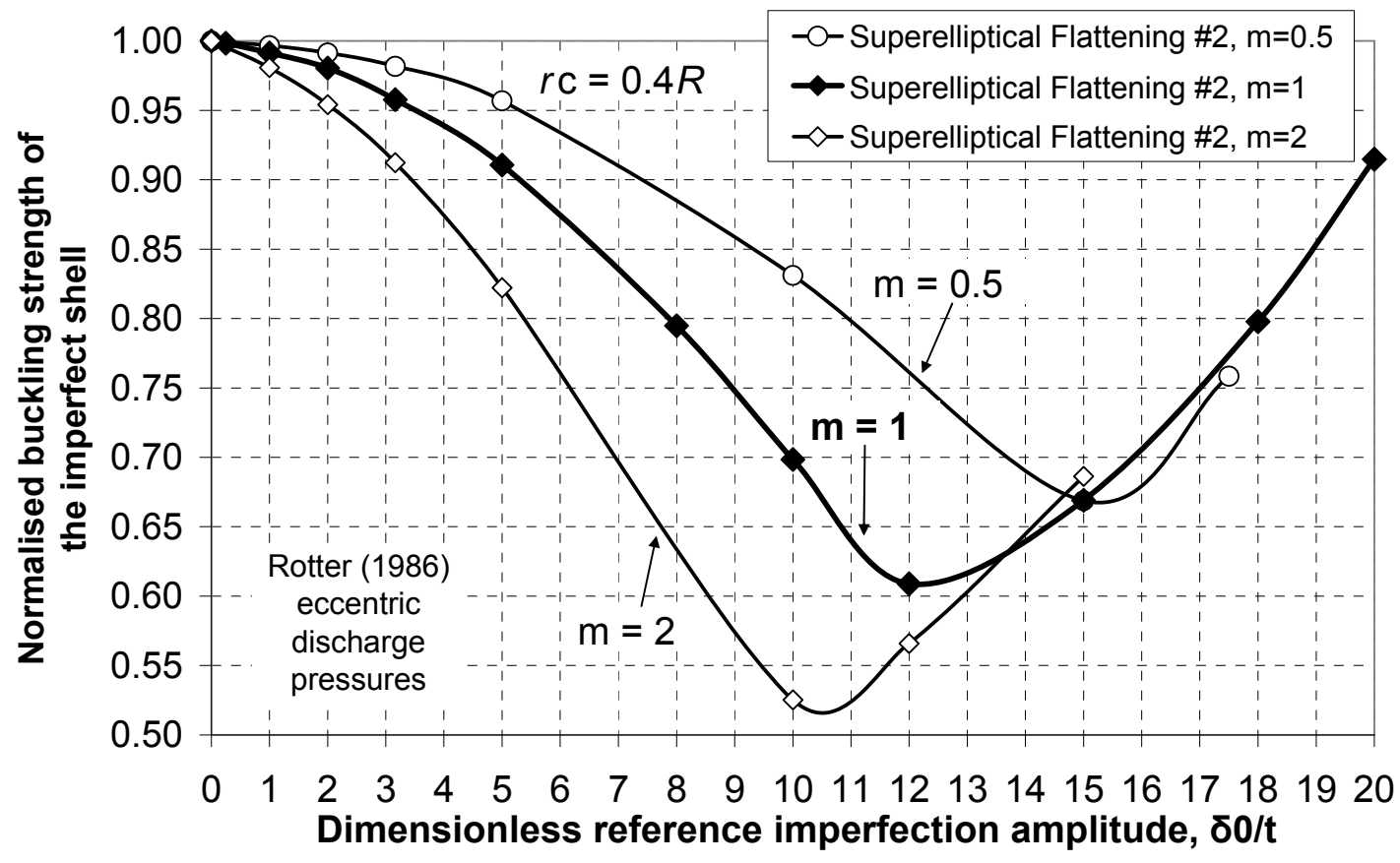

Fig. 19 - Imperfection sensitivity curves for the second superelliptical imperfection form under the Rotter (1986) eccentric discharge pressures with $r_{\mathrm{c}}=0.4 R$

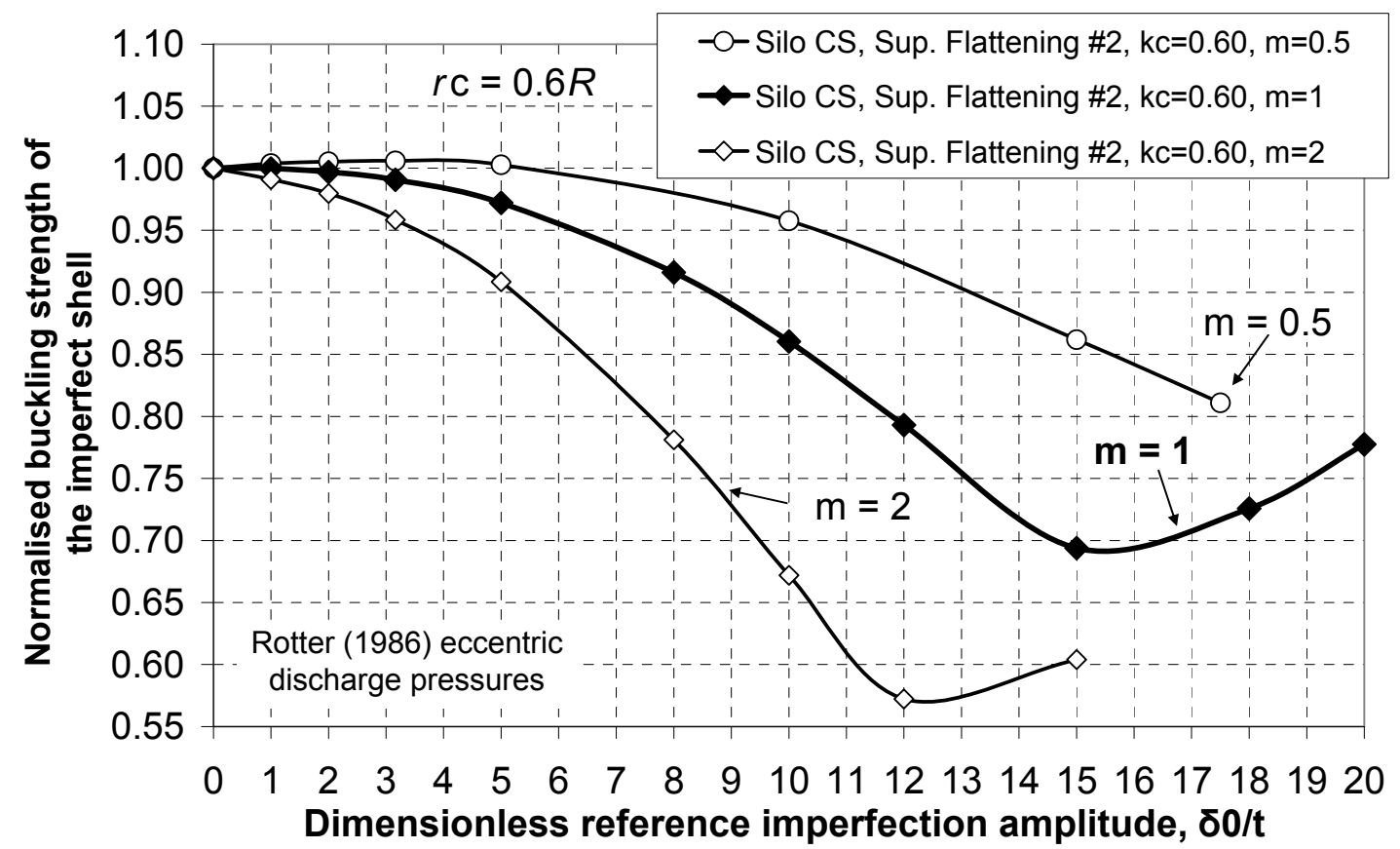

Fig. 20 - Imperfection sensitivity curves for the second superelliptical imperfection form under the Rotter (1986) eccentric discharge pressures with $r_{\mathrm{c}}=0.6 R$ 
Published in: International Journal of Solids and Structures, 50, 781-794.

DOI: http://dx.doi.org/10.1016/j.ijsolstr.2012.11.017

\subsubsection{Imperfection sensitivity study of the second superelliptical imperfection form under the EN 1991-4 (2006) codified eccentric discharge pressures}

The second formulation of the imperfection form was finally analyzed under the official codified eccentric discharge pressures of EN 1991-4 (2006), assuming once again $r_{\mathrm{c}}=0.25 R, 0.4 R$ and $0.6 R$. A single value of $m=2$ was adopted, based on the preceding analyses, since $m \geq 1$ was found to cause greater decreases in the buckling strength. The results are summarized in Fig. 21.

In the analysis of the first formulation (Fig. 16), the buckling strength for the smallest flow channel with $r_{\mathrm{c}}=0.25 R$ was found to increase with $\delta_{0} / t$, which suggested that this imperfection form is not always effective. The second variant of superelliptical flattening shows no such beneficial effect, nor is there any apparent sudden change in the buckling behavior in the range $\delta_{0} \in[0,5 t]$. On this basis, the superelliptical imperfection form with no primary inward feature at the centre of the flow channel may be proposed as the best for the analysis of metal silos under eccentric discharge.

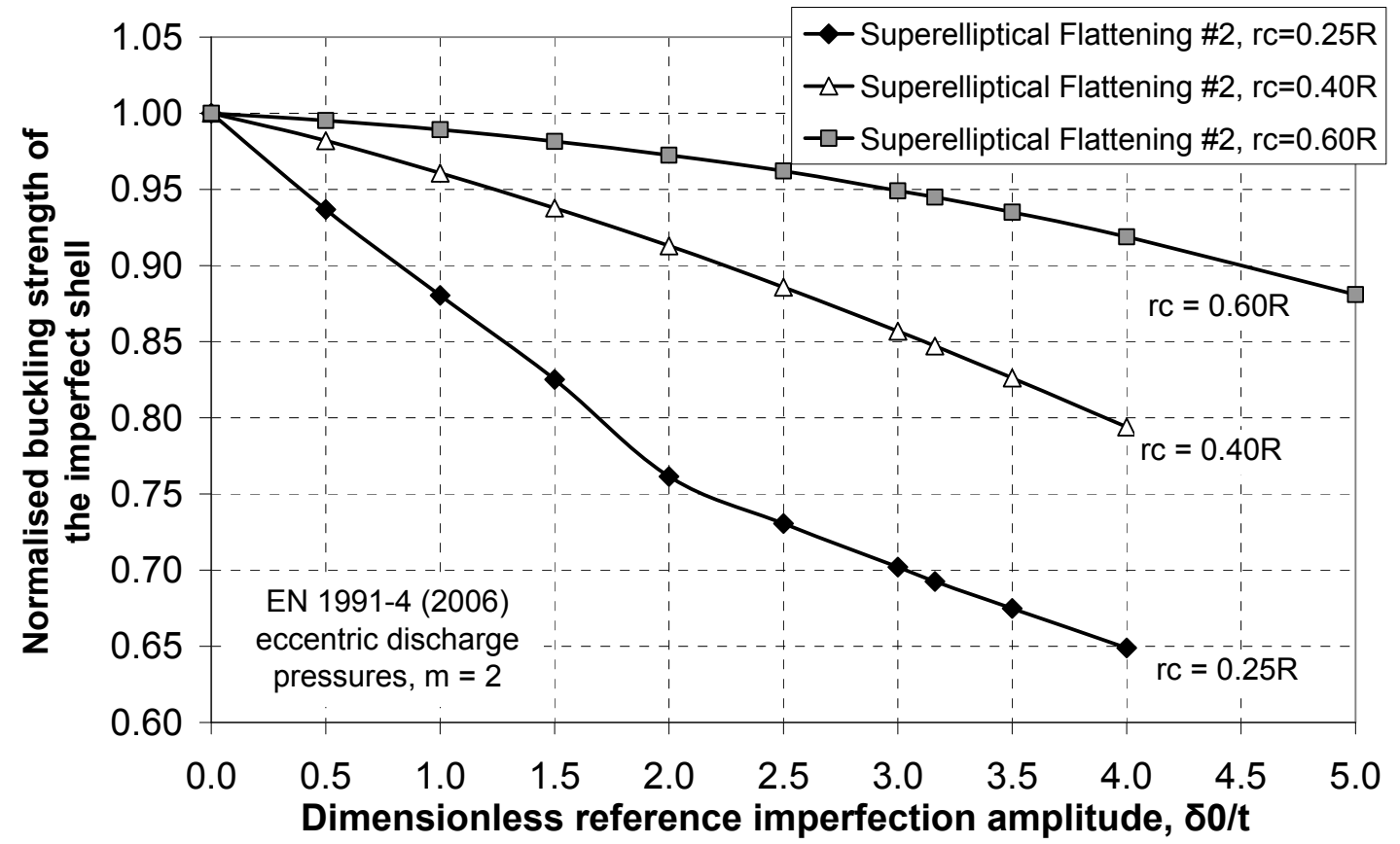

Fig. 21 - Imperfection sensitivity curves for the second superelliptical imperfection form under the EN 1991-4 (2006) codified eccentric discharge pressures 
Published in: International Journal of Solids and Structures, 50, 781-794.

DOI: http://dx.doi.org/10.1016/j.ijsolstr.2012.11.017

\subsubsection{Imperfection sensitivity study of the second superelliptical imperfection form under the EN 1991-4 (2006) codified eccentric discharge pressures on a very slender silo geometry}

A further verification was undertaken to determine whether the deleterious effect of the second superelliptical imperfection form persisted for a thin-walled silo with an aspect ratio higher than $H / D=3$. Eccentric pipe flow is only realistic for slender geometries (Rotter, 2001; Zhong et al., 2001; EN 1991-4, 2006), and as the superelliptical imperfection forms were devised with this flow pattern in mind, they are only proposed here for use in slender silos $(H / D>2)$.

A very slender silo with an aspect ratio $H / D=5.2(H=26 \mathrm{~m}, R=2.5 \mathrm{~m})$ with a stepwise varying wall thickness of $3 \mathrm{~mm}$ to $9 \mathrm{~mm}$ was used for this verification. It was designed in the same manner as the $H / D=3$ silo used above, and further details may be found in Sadowski and Rotter (2012a). This very slender silo was analyzed under the codified eccentric discharge pressures EN 1991-4 (2006) assuming again $r_{\mathrm{c}}=0.25 R, 0.4 R$ and $0.6 R$ and the second superelliptical imperfection form with $m=2$ with amplitudes in the range $\delta_{0} \in[0,4 t]$ (Fig. 22). The results confirm that this imperfection form does indeed cause significant strength losses in a silo with a very high aspect ratio. At $\delta_{0}=2 t$ the reduction in buckling strength is $27 \%$ and $13 \%$ for $r_{\mathrm{c}}=0.4 R$ and $0.6 R$ respectively, compared with only $8 \%$ and $3 \%$ respectively for the silo with $H / D=3$ (Fig. 21). This result indicates that this imperfection form becomes more damaging as the silo slenderness increases. 
Published in: International Journal of Solids and Structures, 50, 781-794.

DOI: http://dx.doi.org/10.1016/j.ijsolstr.2012.11.017

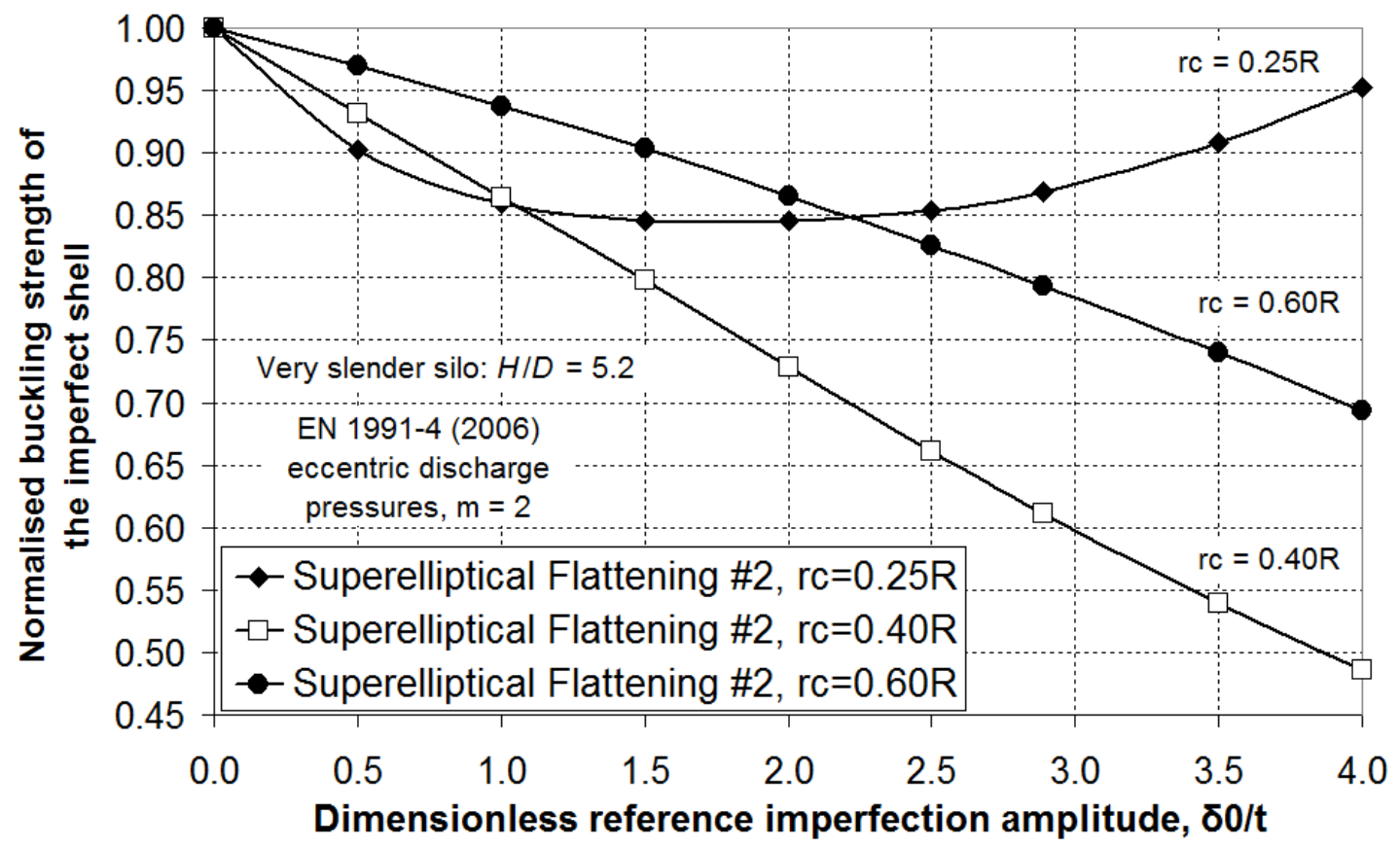

Fig. 22 - Imperfection sensitivity curves for the second superelliptical imperfection form under the EN 1991-4 (2006) codified eccentric discharge pressures on a very slender silo

The imperfection sensitivity curve (Fig. 22) for the smallest channel with $r_{\mathrm{c}}=0.25 R$ is slightly unusual in that a reversal in slope occurs at approximately $\delta_{0}=2 t$. The reason for this is again that the chosen flow channel is too small to induce the global pattern of membrane stresses characteristic of eccentric pipe flow. As a result the superelliptical imperfection form no longer exaggerates the deformations caused by the loading (see discussion on Fig. 16 and Sadowski \& Rotter, 2011b).

However, for design purposes the second superelliptical imperfection may still be considered damaging for $r_{\mathrm{c}}=0.25 R$ because although the relationship becomes beneficial when $\delta_{0}>2 t$. This value is below the EN 1993-1-6 Section 8.7 design amplitude for meridional compression of $2.887 t$ (in a strict interpretation of the standard), but the standard requires (Clause 8.7.2(20)) that the analyst verifies that an imperfection with amplitude $10 \%$ smaller does not produce a lower load factor than the value at full design amplitude. Where this is found to be the case, the analyst must adopt an iterative procedure to locate the minimum of the imperfection sensitivity 
curve. Though a very onerous process, in this case it would lead the analyst to the correct lower strength assessment.

It is evident from Figs 15 to 22 that the "worst" or most realistic unfavorable imperfection forms with their most damaging amplitudes do not reduce the buckling strength below about $60 \%$ of the geometrically nonlinear strength of the perfect shell. This matches the proposal of Rotter (1986), adopted into the Eurocode on silos (EN 1993-4-1, 2007) and the ECCS shell buckling recommendations (Schmidt and Rotter, 2008a) and confirmed by Rotter et al. (2011), that local peaks of axial compression should be designed for systematically higher buckling strengths. The question of what imperfection form and amplitude should be adopted for such a computational assessment is complicated and beyond the scope of this paper, but extended discussions may be found in Rotter (2004) and Schmidt and Rotter (2008b). This paper has confirmed that a search for the most realistic unfavorable imperfection under load conditions of this kind is very challenging, so design rules should probably be based on calculations relating to the perfect shell until more is understood about these phenomena.

\section{Conclusions}

This paper has shown that traditional geometric imperfection forms used to achieve reductions in the buckling strength of shells are not effective for metal silo structures subject to eccentric discharge flows of their contained granular solids. It has then described a search for an appropriately realistic yet deleterious imperfection form that is more appropriate for this loading case, as well as for others involving unsymmetrical loads on stepped wall cylindrical shells. Three potential forms of imperfection have been proposed and explored in the search for suitably damaging imperfections that may be used as a basis for both structural strength and tolerance specifications.

The key findings of the study are:

1. For stepped wall cylindrical shells that buckle under unsymmetrical loads which induce local high axial compressive stresses, the linear bifurcation mode and the nonlinear pre-buckling deformed shape, as well as weld depressions, do not lead 
to imperfections that are deleterious. Advice to computational analysts to use such imperfection forms should be amended to address this problem.

2. The reasons why the geometrically nonlinear pre-buckling deformed shape is not deleterious have been explored and explained.

3. A novel imperfection form, informed by the nonlinear pre-buckling deformed shape, has been proposed using the parametric equation of the superellipse. It has generally produced reliable reductions in buckling strength where the non-linear prebuckling shape failed to do so.

4. This superelliptical imperfection generates the geometry of the entire threedimensional shell explicitly, rather than superimposing a perturbation, and presents a significant change from traditional imperfection definitions. This new approach is not restricted to either the present geometry or load case, but may prove useful for many other shell buckling studies.

5. It has been found that the imperfection sensitivity of the structure, when subject to this load case, displays behavior that is radically different from typical descriptions in the shell buckling literature (e.g. Yamaki, 1984), with a strong sensitivity suddenly replaced by its removal as the imperfection amplitude rises. An appreciation of this behavior is critically important for the practical design of shell structures where tolerance measurements do not necessarily detect the most serious conditions.

6. Eccentric discharge is widely recognized as an extremely damaging phenomenon for thin-walled cylindrical silos, causing buckling at a small fraction of the concentric discharge condition. It is thought that silos under this load condition may be much less imperfection sensitive than uniformly compressed cylinders (Rotter, 1986), and it is suggested that computational design analyses of silos under eccentric discharge should perhaps be undertaken using only the perfect geometry. 
Published in: International Journal of Solids and Structures, 50, 781-794.

DOI: http://dx.doi.org/10.1016/j.ijsolstr.2012.11.017

\section{References}

ABAQUS (2009). “ABAQUS Version 6.9” Dassault Systèmes Simulia Corp., Providence, RI, USA. www.simulia.com

Blachut J., Galletly G.D. \& Moreton D.N. (1990). "Buckling of Near-Perfect Steel Torispherical and Hemispherical Shells subjected to external pressure." AIAA Journal, Vol. 28, N. 11, November, 1971-1975.

Blachut J. \& Galletly G.D. (1987). "Externally pressurised hemispheres and shallow torispheres." Proc. Int. Colloquium on the Stability of Plate and Shell Structures, Gent, Belgium, 6-8 April, ECCS, 361-366.

Blachut J. \& Galletly G.D. (1990). "Buckling Strength of Imperfect Spherical Caps Some Remarks.” AIAA Journal, Vol. 28, N. 7, July, 1314-1319.

Chen J.F., Rotter J.M., Ooi J.Y. \& Zhong Z. (2007). "Correlation between the flow pattern and wall pressures in a full scale silo.” Engineering Structures, 29, 2308-2320.

EN 1991-4 (2006). "Eurocode 1 - Actions on structures. Part 4: Silos and tanks." European Committee for Standardization, Brussels.

EN 1993-1-6 (2007). "Eurocode 3: Design of Steel Structures, Part 1-6: Strength and Stability of Shell Structures." Comité Européen de Normalisation, Brussels.

EN 1993-4-1 (2007). "Eurocode 3: Design of Steel Structures, Part 4-1: Silos." Comité Européen de Normalisation, Brussels.

Esslinger M. \& Geier B. (1972). “Gerechnete Nachbeulasten als untere Grenze der experimentellen axialen Beulasten von Kreiszylindern.” Der Stahlbau, 41(12), 353360.

Gale, B.R., Hoadley, P.J. and Schmidt, L.C., "Aspects of Eccentric Discharge of Granular Material from a Circular Silo”, Proc., Second International Conference on 
Published in: International Journal of Solids and Structures, 50, 781-794.

DOI: http://dx.doi.org/10.1016/j.ijsolstr.2012.11.017

Bulk Materials Storage Handling and Transportation, Institution of Engineers, Australia, Wollongong, July 1986, pp 258-263.

Gridgeman N. T. (1970). “Lamé ovals.” Math. Gaz., 54, 31-37.

Guggenberger W., Greiner R \& Rotter J.M. (2004). "Cylindrical shells above local supports." Chapter 3 in Buckling of Thin Metal Shells, eds J.G. Teng \& J.M. Rotter, Spon, London, pp 88-128.

Hampe E. (1991). "Silos: Band 1 Grundlagen: Band 2 Bauwerke." Verlag für Bauwesen GmbH, Berlin.

Hutchinson, J.W., Tennyson, R.C. and Muggeridge, D.B. (1971) "Effect of a Local Axisymmetric Imperfection on the Buckling Behaviour of a Circular Cylindrical Shell under Axial Compression", American Institute of Aeronautics and Astronautics Jnl, Vol. 9, No. 1, Jan., pp 48-53.

Jenike A.W. (1967). "Denting of Circular Bins with Eccentric Drawpoints.” J. of the Sruct. Div., ASCE, 93 (ST1) 27-35.

Knödel, P., Ummenhofer, T. \& Schulz, U. (1995). "On the Modelling of Different Types of Imperfections in Silo Shells.” Thin-Walled Structures, 23, 283-293.

Koiter W.T. (1945). "On the stability of elastic equilibrium.” PhD Thesis, Delft University, Holland.

Koiter W.T. (1963). "The effect of axisymmetric imperfections on the buckling of cylindrical shells under axial compression." Proc. Kon. Ned. Akad. Wet., B66, 265279. (See also Appl. Mech. Rev., 18, Review 3387, 1965).

Lamé G. (1818). "Examen des différentes méthodes employées pour résoudre les problèmes de géométrie.” Vve Courcier Imprimeur-Libraire, Paris. 
Published in: International Journal of Solids and Structures, 50, 781-794.

DOI: http://dx.doi.org/10.1016/j.ijsolstr.2012.11.017

Libai A. \& Durban D. (1973). "Buckling of cylindrical shells subjected to nonuniform axial loads.” Jrnl. of Applied Mech, 44, Trans ASME, 714-720.

Nielsen, J. (1998) "Pressures from flowing granular solids in silos", Phil. Trans. Royal Society of London: Philosophical Transactions: Mathematical, Physical and Engineering Sciences, Series A, Vol. 356, No. 1747, pp 2667-2684.

Rotter J.M. (1985). “Design under axial compression.” Design of Steel Bins for the Storage of Bulk Solids, ed. J.M. Rotter, The University of Sydney, Australia, 122-137.

Rotter J.M. (1986). "The analysis of steel bins subject to eccentric discharge." Proc. of the $2^{\text {nd }}$ Int. Conf. on Bulk Materials Storage, Handling and Transportation, IEAustralia, Wollongong, 264-271.

Rotter J.M. (1989). "Stress Amplification in Unstiffened Cylindrical Steel Silos and Tanks." Civil Eng. Transactions, IE Aust, CE31(3), 142-148.

Rotter J.M. (2001a). "Guide for the economic design of circular metal silos.” Spon Press, London.

Rotter, J.M. (2001b). "Pressures, Stresses and Buckling in Metal Silos containing Eccentrically Discharging Solids", Festschrift Richard Greiner, Celebration volume for the 60th birthday of Prof. Richard Greiner, TU Graz, Austria, October pp 85-104.

Rotter J.M. (2004). "Buckling of cylindrical shells under axial compression." Chapters 1 \& 2 in Buckling of Thin Metal Shells, Eds J.G. Teng \& J.M. Rotter, Spon, London, pp 1-87.

Rotter J.M. \& Teng J.G. (1989). "Elastic stability of cylindrical shells with weld depressions." Jrnl. of Struct. Eng., ASCE, 115(5), 1244-1263.

Rotter J.M. \& Zhang Q. (1990). "Elastic buckling of imperfect cylinders containing granular solids.” Jrnl. of Struct. Eng., ASCE, 116(8), 2253-2271. 
Published in: International Journal of Solids and Structures, 50, 781-794.

DOI: http://dx.doi.org/10.1016/j.ijsolstr.2012.11.017

Rotter, J.M., Cai, M. and Holst, J.M.F.G. (2011) "Buckling of thin cylindrical shells under locally elevated axial compression stresses", Journal of Pressure Vessel Technology, 133(1), 11204-1 to 11.

Sadowski A.J. (2010). "Modeling of failures in thin-walled metal silos under eccentric discharge." PhD Thesis, The University of Edinburgh.

Sadowski A.J. (2011). "Geometric properties for the design of unusual member crosssections in bending." Engineering Structures, 33(5), 1850-1854.

Sadowski A.J. \& Rotter J.M. (2010). “A Study of Buckling in Steel Silos under Eccentric Discharge Flows of Stored Solids.” Jrnl. of Eng. Mech., ASCE, 136(6), 769-776.

Sadowski A.J. \& Rotter J.M. (2011a). "Buckling of very slender metal silos under eccentric discharge." Engineering Structures, 33(4), 1187-1194.

Sadowski A.J. \& Rotter J.M. (2011b). "Steel silos with different aspect ratios: II behavior under eccentric discharge." Journal of Constructional Steel Research, 67(10), 1545-1553.

Sadowski A.J. \& Rotter J.M. (2012a). "The structural behavior of thin-walled metal silos subject to different flow channel sizes under eccentric discharge pressures." ASCE Journal of Structural Engineering, 138(7), 922-931.

Sadowski A.J. \& Rotter J.M. (2012b). "Buckling in eccentrically discharged silos and the assumed pressure distribution." Accepted for publication, ASCE Journal of Engineering Mechanics. http://dx.doi.org/10.1061/(ASCE)EM.1943-7889.0000525

Schmidt, H. \& Rotter J.M. (2008a) "Cylindrical shells of constant wall thickness under general loading" Chapter 10 in Stability of Steel Shells: European Design Recommendations: Fifth Edition, European Convention for Constructional Steelwork, Brussels, pp 167-216. 
Published in: International Journal of Solids and Structures, 50, 781-794.

DOI: http://dx.doi.org/10.1016/j.ijsolstr.2012.11.017

Schmidt, H. \& Rotter J.M. (2008b) "Rules for the buckling limit state assessment using global numerical analysis" Chapter 8 in Stability of Steel Shells: European Design Recommendations: Fifth Edition, European Convention for Constructional Steelwork, Brussels, pp 95-145.

Song C.Y., Teng J.G. \& Rotter J.M. (2004). "Imperfection sensitivity of thin elastic cylindrical shells subject to partial axial compression." Int. Jrnl. of Solids and Structures, 41, 7155-7180.

Wallner, S. (2010a) "Neue Regelungen gem. Eurocode (EN 1991-4 und EN 1993-4-1) zur Berechnung von Stahlsilos”, Section 5, Stahlbaukalender 2010, Verlag Ernst und Sohn, Berlin.

Wallner, S. (2010b) "Neue Regelungen gemäß Eurocode (EN 1991-4 und EN 1993-41) zur Berechnung von Stahlsilos - Darstellung anhand eines Schadensfalles an einem diskret gelagerten Schraubsilo", Biberach Vortrag, Wien.

Yamaki N. (1984). "Elastic Stability of Circular Cylindrical Shells." North-Holland, Amsterdam.

Zhong Z., Ooi J.Y. \& Rotter J.M. (2001). "The sensitivity of silo flow and wall stresses to filling method." Engineering Structures, 23, 756-767. 